This article has been accepted for publication in Monthly Notices of the Royal Astronomical Society (C: 2021 The Authors. Published by Oxford University Press on behalf of the Royal Astronomical Society. All rights reserved. 


\title{
Hard X-ray selected giant radio galaxies - III. The LOFAR view
}

\author{
G. Bruni ${ }^{\circledR},{ }^{1 \star}$ M. Brienza, ${ }^{2,3}$ F. Panessa ${ }^{\circledR},{ }^{1}$ L. Bassani, ${ }^{4}$ D. Dallacasa, ${ }^{2,3}$ T. Venturi, ${ }^{3}$ R. D. Baldi ${ }^{\circledR},{ }^{3,5}$
} A. Botteon ${ }^{\oplus},{ }^{6}$ A. Drabent, ${ }^{7}$ A. Malizia, ${ }^{4}$ F. Massaro,,${ }^{8,9,10,11}$ H. J. A. Röttgering, ${ }^{6}$ P. Ubertini, ${ }^{1}$ F. Ursini ${ }^{\oplus 12}$ and R. J. van Weeren ${ }^{6}$

${ }^{1}$ INAF - Istituto di Astrofisica e Planetologia Spaziali, via Fosso del Cavaliere 100, I-00133 Roma, Italy

${ }^{2}$ DiFA - Dipartimento di Fisica e Astronomia, Università di Bologna, via Piero Gobetti 93/2, I-40129 Bologna, Italy

${ }^{3}$ INAF - Istituto di Radioastronomia, via Piero Gobetti 101, I-40129 Bologna, Italy

${ }^{4}$ INAF - Osservatorio di Astrofisica e Scienza dello Spazio di Bologna, via Piero Gobetti 93/3, I-40129 Bologna, Italy

${ }^{5}$ School of Physics and Astronomy, University of Southampton, Southampton SO17 1BJ, UK

${ }^{6}$ Leiden Observatory, Leiden University, PO Box 9513, NL-2300 RA Leiden, the Netherlands

${ }^{7}$ Thüringer Landessternwarte, Sternwarte 5, D-07778 Tautenburg, Germany

${ }^{8}$ Dipartimento di Fisica, Università degli Studi di Torino, via Pietro Giuria 1, I-10125 Torino, Italy

${ }^{9}$ INAF - Osservatorio Astrofisico di Torino, via Osservatorio 20, I-10025 Pino Torinese, Italy

${ }^{10}$ Istituto Nazionale di Fisica Nucleare, Sezione di Torino, I-10125 Torino, Italy

${ }^{11}$ Consorzio Interuniversitario per la Fisica Spaziale, via Pietro Giuria 1, I-10125 Torino, Italy

${ }^{12}$ Dipartimento di Matematica e Fisica, Università degli Studi Roma Tre, via della Vasca Navale 84, I-00146 Roma, Italy

Accepted 2021 February 23. Received 2021 February 18; in original form 2020 December 29

\section{ABSTRACT}

Giant radio galaxies (GRGs), with extended structures reaching hundreds of kpc, are among the most spectacular examples of ejection of relativistic plasma from supermassive black holes. In this work, third of a series, we present Low-Frequency Array (LOFAR) images at $144 \mathrm{MHz}$, collected in the framework of the LOFAR Two-metre Sky Survey Data Release 2 (LoTSS DR2), for nine sources extracted from our sample of hard X-ray-selected GRGs (HXGRGs, i.e. from INTEGRAL/Imager on-Board the INTEGRAL Satellite and Swift/Burst Alert Telescope catalogues at $>20 \mathrm{keV}$ ). Thanks to the resolution and sensitivity of LoTSS, we could probe the complex morphology of these GRGs, unveiling cases with diffuse (Mpc-scale) remnant emission, presence of faint off-axis wings, or a misaligned inner jet. In particular, for one source (B2 1144+35B), we could clearly detect a $\sim 300 \mathrm{kpc}$ wide off-axis emission, in addition to an inner jet whose orientation is not aligned with the lobes axis. For another source (J1153.9+5848), a structure consistent with jet precession was revealed, appearing as an X-shaped morphology with relic lobes having an extension larger than the present ones, and with a different axis orientation. From an environment analysis, we found two sources showing an overdensity of cosmological neighbours, and a correspondent association with a galaxy cluster from catalogues. Finally, a comparison with radio-selected GRGs from LoTSS DR1 suggested that, on average, HXGRGs can grow to larger extents. These results highlight the importance of deep low-frequency observations to probe the evolution of radio galaxies, and ultimately estimate the duty cycle of their jets.

Key words: galaxies: active-galaxies: jets - galaxies: nuclei - galaxies: Seyfert-radio continuum: galaxies-X-rays: galaxies.

\section{INTRODUCTION}

Giant radio galaxies (GRGs; Willis, Strom \& Wilson 1974) are among the most extreme manifestations of the accretion/ejection processes on supermassive black holes. During their $\sim 100$ Myr timescale evolution (Machalski, Chyzy \& Jamrozy 2004; Jamrozy et al. 2008), they produce jets of plasma extending hundreds of kpc away from their active galactic nucleus (AGN) core. Their projected linear size, comprised between a conventional threshold of $0.7 \mathrm{Mpc}$ and a maximum of $4.69 \mathrm{Mpc}$ to date (Machalski et al. 2008), implies that their jets are able to probe the intergalactic medium, at spatial scales comparable with galaxy clusters. In the past decades, several authors performed detailed studies of the age of GRGs, on the basis

^E-mail: gabriele.bruni@inaf.it of their kinematics or plasma radiative losses, dating the ancient lobes as structures formed during tens, if not hundreds, of Myr (see e.g. Mack et al. 1998; Hunik \& Jamrozy 2016; Cantwell et al. 2020), eventually becoming remnant radio structures (Hurley-Walker et al. 2015; Brienza et al. 2016). With such a long activity period, GRGs are the ideal testbed to study the duration of the radio phase in AGN, and its duty cycle. Indeed, several examples of GRGs with recurrent radio activity have been found by different authors (Konar et al. 2004; Orrù et al. 2015; Hunik \& Jamrozy 2016; Bruni et al. 2020), either in the form of two symmetric pairs of lobes with respect to the active nucleus (termed double-double radio galaxy; Schoenmakers et al. 2000), or an X-shaped morphology (misaligned double-double; Cheung 2007; Saripalli et al. 2013) possibly linked to a change in jet axis, or the presence of a young radio source in the core at odds with the age inferred from the Mpc-scale lobes (Bruni et al. 2019). 
With the advent of the new generation low-frequency surveys the number of known GRGs has dramatically increased, populating the parameter space of GRGs. This suggests that their abundance has been underestimated so far, mainly because of the limited sensitivity to extended emission of the previous generation radio surveys (Delhaize et al. 2021). In particular, Low-Frequency Array (LOFAR; van Haarlem et al. 2013), with its unique sensitivity to extended steep-spectrum structures and a typical resolution of 6 arcsec in the $\mathrm{MHz}$ domain, is the ideal instrument to study these complex and low surface brightness objects. Indeed, the emission from plasma generated at the beginning of GRGs activity shows steep spectra, and therefore its emission is brighter and more easily detected at low frequencies. In the framework of the LOFAR surveys key science project, single objects (Shulevski et al. 2019; Cantwell et al. 2020) and samples of GRGs (Dabhade et al. 2020a; Stuardi et al. 2020) have been studied with unprecedented detail, allowing to extend the census to hundreds of sources. As a whole, morphological studies at low frequencies, through synthesis imaging with radio interferometers, can help understanding the plasma dynamics. This information can then be used to investigate the duty cycle of the radio phase in AGN.

In the past few years, the Giant RAdio galaxies and their duty CyclE (GRACE) ${ }^{1}$ project has targeted GRGs selected by crosscorrelating hard X-ray catalogues $(>20 \mathrm{keV})$ from Swift/Burst Alert Telescope (BAT) and INTEGRAL/Imager on-Board the INTEGRAL Satellite (IBIS) with radio catalogues (the NRAO Very Large Array Sky Survey - NVSS, Condon et al. 1998 - and the Sydney University Molonglo Sky Survey - SUMSS, Bock, Large \& Sadler 1999). As a first result of this selection, Bassani et al. (2016) found that the fraction of GRGs in the parent sample of 64 radio galaxies were about four times larger than in previous radio-only studies ( $\sim 25$ per cent versus the classical $\sim 6$ per cent from the $3 \mathrm{CR}$ catalogue, see Ishwara-Chandra \& Saikia 1999, and 8 per cent in the 3CRR sample, see Laing, Riley \& Longair 1983). We thus embarked on a multiwavelength study of the 15 GRGs from the Bassani et al. (2016) sample (see Bruni et al. 2020 for the full sample description), aimed at understanding the reason for such a correlation. In the first paper of this collection, Ursini et al. (2018) showed how the radio luminosity of the lobes and jet power are dimmer than what expected from the X-ray power of the core, suggesting a recently restarted activity. This was later on confirmed by Bruni et al. $(2019,2020)$, either on the basis of the radio morphology, or for the presence of a recently restarted radio source in the core.

In this paper, for the first time, we present LOFAR images of nine hard X-ray-selected giant radio galaxies (HXGRGs), aiming at characterizing their large-scale morphology and signs of restarted radio activity. Moreover, we perform a comparison between HXGRGs and a sample of radio-selected GRGs to investigate systematic differences in their main properties. Recently, Bassani et al. (2021) presented an update of the sample, adding eight GRGs plus a candidate source. We present here five GRGs from the original sample, plus another four from the catalogue update. In the following, we adopt the convention $S \propto v^{\alpha}$ for the spectral index $\alpha$, and the same cosmological parameters as in Bassani et al. (2016) for consistency, i.e. $H_{0}=71 \mathrm{~km} \mathrm{~s}^{-1} \mathrm{Mpc}^{-1}, \Omega_{\mathrm{m}}=0.27$, and $\Omega_{\Lambda}=0.73$.

\footnotetext{
${ }^{1}$ https://sites.google.com/inaf.it/grace/
}

\section{RADIO DATA}

\subsection{LOFAR}

We made use of data from the LOFAR Two-metre Sky Survey (LoTSS; Shimwell et al. 2017) Data Release 1 and the upcoming Data Release 2 (DR1 and DR2 hereafter), carried out at $144 \mathrm{MHz}$ (frequency range 120-168 MHz). LoTSS DR1 (Shimwell et al. 2019) covered an area of $424 \mathrm{deg}^{2}$ in the Hobby-Eberly Telescope Dark Energy Experiment (HETDEX) spring field, between right ascension (RA) $10^{\mathrm{h}} 45^{\mathrm{m}} 00^{\mathrm{s}}$ to $15^{\mathrm{h}} 30^{\mathrm{m}} 00^{\mathrm{s}}$ and declination (Dec.) $45^{\circ} 00^{\prime} 00^{\prime \prime}$ to $57^{\circ} 00^{\prime} 00^{\prime \prime}$, reaching a median sensitivity of $71 \mu \mathrm{Jy} \mathrm{beam}^{-1}$ at 6 arcsec angular resolution. LoTSS DR2 (Shimwell et al., in preparation) will offer additional coverage on two fields centred at a Galactic latitude of $0^{\mathrm{h}}$ and $13^{\mathrm{h}}$, for a total of $5700 \mathrm{deg}^{2}$, and processed with an improved version of the pipeline. Among the nine sources analysed in this work, only one (J1238.4+5349) is located in the HETDEX region, and therefore was already published as part of the LoTSS DR1, while the remaining eight are located in the sky region covered by the DR2, and are presented here for the first time. All data sets used in this work have been processed by the Survey KSP team using the standard DR2 approach (Shimwell et al., in preparation). This includes the use of an upgraded version of the LoTSS direction-dependent pipeline (Tasse et al. 2018, 2020), which leads to significant improvements of the final images with respect to the DR1.

To further refine the imaging in the direction of the nine targets, we proceeded as follows. First, we subtracted out of the visibilities all the sources outside the region containing the target, and performed additional phase and amplitude self-calibration loops in the target direction, following the same procedure as previous works from the LoTSS Collaboration (van Weeren et al. 2020). We then used these data sets to produce the final images running the WSCLEAN imager (Offringa et al. 2014) on the Bologna node of the LOFAR-IT infrastructure, fine-tuning different sets of parameters with the goal of enhancing the reconstruction of the diffuse and faint emission of the sources. Final images were obtained with two circular Gaussian restoring beams of 6 and 20 arcsec for each source, to exploit both the full resolution of the array and a tailored large beam to better detect the diffuse emission. For both resolutions we used a Briggs weighting scheme with robust $=-0.5$ and multiscale cleaning (see Offringa \& Smirnov 2017). Additionally, we applied a Gaussian taper to obtain 20 arcsec angular resolution images.

Table 1 summarizes the final typical rms noise obtained for the different images, at both resolutions, and for each source. Analogously to Shimwell et al. (2019), and to recent works on GRGs performed with LoTSS data (Dabhade et al. 2020a), we assumed a conservative error of 20 per cent for the measured flux densities.

Table 1. Summary of LoTSS data presented in this work, with typical rms noise obtained for the images convolved at 6 and 20 arcsec resolution.

\begin{tabular}{lcc}
\hline Source & $\begin{array}{r}\text { rms }(6 \text { arcsec }) \\
\left(\mu \mathrm{Jy} \mathrm{beam}^{-1}\right)\end{array}$ & $\left.\begin{array}{r}\text { rms }(20 \text { arcsec }) \\
(\mu \mathrm{Jy} \mathrm{beam}\end{array}\right)$ \\
\hline J0318+684 & 220 & 360 \\
J0801.7+4764 & 130 & 420 \\
B2 1144+35B & 90 & 180 \\
J1153.9+5848 & 100 & 160 \\
J1238.4+5349 & 90 & 240 \\
J1503.7+6850 & 150 & 390 \\
4C +63.22 & 120 & 360 \\
Mrk 1498 & 110 & 220 \\
4C +34.47 & 230 & 760 \\
\hline
\end{tabular}


Table 2. Summary of VLA data presented in this work, from our observations (20A-476) and archives (15B-145).

\begin{tabular}{lcccc}
\hline Source & Code & $\begin{array}{c}\text { Frequency } \\
(\mathrm{GHz})\end{array}$ & $\begin{array}{c}\text { Beam } \\
(\operatorname{arcsec})\end{array}$ & $\begin{array}{c}\mathrm{rms} \\
\left(\mu \mathrm{Jy} \mathrm{beam}{ }^{-1}\right)\end{array}$ \\
\hline J0318+684 & 20A-476 & 3 & 20 & 200 \\
Mrk 1498 & 15B-145 & 6 & $35 \times 15$ & 60 \\
\hline
\end{tabular}

\subsection{VLA}

For two sources (J0318+684 and Mrk 1498), we could consider additional observations from the Karl G. Jansky Very Large Array (VLA, see Table 2). For J0318+684, Director's Discretionary Time (DDT) observations were obtained, and performed on 2020 May 24 (project 20A-476) with the array in C-configuration at $3 \mathrm{GHz}$. The on-source time was $15 \mathrm{~min}$. For Mrk 1498, we made use of archival data (project 15B-145). Observations at $6 \mathrm{GHz}$ were performed on 2015 November 11 in D-configuration, for a total on-source time of $36 \mathrm{~min}$.

Data were reduced with the $\mathrm{CASA}^{2}$ software (McMullin et al. 2007), running VLA pipeline for initial calibration. Then, target data were split and imaging performed with the TCLEAN task, through two iterations of phase-only self-calibration. For J0318+684, almost 40 per cent of the bandwidth was flagged out for the $3 \mathrm{GHz}$ observations, due to radio frequency interference. A final rms noise of $200 \mu \mathrm{Jy}_{\text {beam }}{ }^{-1}$ was obtained. For Mrk 1498, in order to mitigate the lack of sensitivity of the VLA to structures larger than 4 arcmin (in D configuration and at $6 \mathrm{GHz}$ ), we made use of the lower 25 per cent of the receiver band only (4-5 GHz), recovering as much as possible the extended emission of the target. This resulted in a final rms noise of $60 \mu \mathrm{Jy}$ beam $^{-1}$.

\section{RESULTS}

In the following, we present the morphology revealed by LOFAR images for nine HXGRGs extracted from Bassani et al. (2016) or Bassani et al. (2021). In the figures, the 6 arcsec resolution image is plotted in colour scale, while the contours are from the 20 arcsec resolution one, unless specified differently. For a few sources, we also provide a zoom of the core region. The X-ray position of the core from Bruni et al. (2020) or Bassani et al. (2021) is indicated with a cross. In Table 3, we report quantities extracted from LOFAR images, including updated values for the projected linear sizes (estimated from the angular size between the outermost $3 \times$ rms contours) and total flux densities from the LOFAR 20 arcsec resolution images.

\section{$3.1 \mathrm{~J} 0318+684$}

This is one of the most extended GRGs of our sample (1.5 Mpc; Bassani et al. 2016). In our previous works, we discussed the discovery of a young radio source (gigahertz-peaked spectrum - GPS - source) in the core region, suggesting a recent reactivation of the jet (Bruni et al. 2019), and discussed the X-shaped like morphology (Bruni et al. 2020). Beyond our proprietary data, in the discussion of this object we considered other data available in the literature. Namely, Very Large Array Sky Survey (VLASS) data at $3 \mathrm{GHz}$ (first epoch quick-look image; Lacy et al. 2020), and optical images from the Panoramic Survey Telescope and Rapid Response System (Pan-STARRS; Chambers et al. 2016).

\footnotetext{
${ }^{2}$ https://casa.nrao.edu/
}

In Fig. 1 (top panel), we show the LOFAR full resolution image of the source, with the low-resolution image contours overlaid. The source morphology is consistent with our Giant Metrewave Radio Telescope (GMRT) image at $325 \mathrm{MHz}$ from Bruni et al. (2020). The northern and southern lobes are the brightest components, with the latter having a peak surface brightness of $143 \mathrm{mJy}^{\text {beam }}{ }^{-1}$ with respect to $17.3 \mathrm{mJy}^{\text {beam }}{ }^{-1}$ for the northern one (at 6 arcsec resolution). The core is well visible as a compact and bright-spot at the centre of the source, with a peak of $13.1 \mathrm{mJy}^{\text {beam }}{ }^{-1}$. Diffuse, offaxis emission is present, with a remarkable extension of $\sim 3$ arcmin towards north-west (NW) and $\sim 2.5$ arcmin south-east (SE) from the core (corresponding to 300 and $250 \mathrm{kpc}$, respectively). Overall, more diffuse emission between core and lobes is visible with respect to our previous GMRT observations. Zoomed images of the lobes and core regions are reported in Fig. 1 (bottom panels). Both the northern and southern lobes present a hotspot, typical of the Faranoff-Riley type II (FRII) morphology, with an enhanced surface brightness due to the interaction of the jet plasma with the intergalactic medium (IGM). This is a clear sign of a recent replenishment of plasma, indicating that the jets are still active or, from kinematic considerations, that the radio phase fuelling the lobes has stopped a maximum of $T<$ $\mathrm{LS} / 2 / v$ kyr ago, where $v$ is the average jet velocity (typically $\sim 0.1 c$ ), and LS is the linear size of the GRG in kpc $(1500 \mathrm{kpc})$. This roughly correspond to a $T<24 \mathrm{Myr}$. The southern lobe shows a knotty structure in the region closest to the edge $(\sim 100 \mathrm{kpc}$, see Fig. 1 , bottom right-hand panel). The off-axis extended emission, already highlighted by our GMRT map at $325 \mathrm{MHz}$ from Bruni et al. (2020), is even more evident in the LOFAR image, as expected from the very steep $(<-1)$ spectral index discussed in our previous work.

In Fig. 2 (left-hand panel), we show the comparison between LOFAR (in colours) and our VLA image ( $3 \mathrm{GHz}$, contours), restored with a matched angular resolution of 20 arcsec: the off-axis emission is only partially detected by the VLA (due to its lower sensitivity to extended structures, and the steep-spectral index), while the southern jet/lobe and the core are evident. Analogously to the LOFAR image, the northern lobe is fainter with respect to the southern one. The first half of the structure connecting the core to the northern lobe is not detected. Finally, observations at 6 and $10 \mathrm{GHz}$ were designed to image the core region, which was detected as a compact component. However, the limited resolution and ultraviolet (UV) coverage of these short DDT observations did not allow us to resolve the core at the kpc scale, we thus make use of VLASS data for this purpose (see below). In Fig. 2 (right-hand panel), we present a zoom into the core region, making use of the Pan-STARRS $i$-band image (in grey), tracing the host galaxy structure, and VLASS data (contours), showing the radio emission with better detail than our VLA observations. Indeed, at an angular resolution of $3 \times 2 \operatorname{arcsec}^{2}$, VLASS contours allow us to see an elongation towards the southwest direction, suggesting an inner jet orientation compatible with the Mpc-scale jet one. The new radio phase, previously identified through a GPS, has thus most probably the same jet axis as the older one. At the redshift of the source, the deconvolved size of the radio core as measured from the VLASS image is $0.9 \times 0.4 \operatorname{arcsec}^{2}$, at a position angle (PA) of $\sim 50^{\circ}$, corresponding to a projected linear size of $1.5 \times 0.7 \mathrm{kpc}^{2}$. This size is compatible with the one expected for GPS sources ( $\leq 1 \mathrm{kpc}$; O'Dea 1998). This is also confirmed by archival Very Long Baseline Array (VLBA) observations at pc-scale resolution (Bruni et al., in preparation).

In a recent work presenting MeerKAT observations, Cotton et al. (2020) have shown hydrodynamical backflow in action in a GRG in common with our sample (PKS 2014-55; see Bruni et al. 2020), underlying how the host galaxy PA, and thus its interstellar medium, 
Table 3. Quantities for the sample of nine HXGRGs presented in this work, including projected linear sizes, total flux densities, and total radio power, as measured from the LOFAR images at $144 \mathrm{MHz}$ (20 arcsec resolution).

\begin{tabular}{lccccccr}
\hline Source & $\begin{array}{c}\text { RA } \\
(\mathrm{J} 2000)\end{array}$ & $\begin{array}{c}\text { Dec. } \\
(\mathrm{J} 2000)\end{array}$ & $z$ & $\begin{array}{c}\text { Spatial scale } \\
\left(\mathrm{kpc} \mathrm{arcsec}^{-1}\right)\end{array}$ & $\begin{array}{c}\text { LS } \\
(\mathrm{kpc})\end{array}$ & $\begin{array}{c}\text { Total flux density } \\
(\mathrm{Jy})\end{array}$ & $\begin{array}{c}\text { Total radio power } \\
\log \left(\mathrm{W} \mathrm{Hz} \mathrm{Hz}^{-1}\right)\end{array}$ \\
\hline J0318+684 & $03: 18: 19.1$ & $+68: 29: 32$ & 0.09 & 1.659 & 1620 & $5.67 \pm 1.13$ & 26.03 \\
J0801.7+4764 & $08: 01: 31.9$ & $+47: 36: 15$ & 0.16 & 2.729 & 1260 & $0.46 \pm 0.09$ & 25.47 \\
B2 1144+35B & $11: 47: 22.3$ & $+35: 01: 09$ & 0.06 & 1.144 & 860 & $1.46 \pm 0.29$ & 25.08 \\
J1153.9+5848 & $11: 53: 23.9$ & $+58: 31: 41$ & 0.20 & 3.268 & 900 & $0.26 \pm 0.05$ & 25.43 \\
J1238.4+5349 & $12: 38: 07.7$ & $+53: 25: 56$ & 0.35 & 4.907 & 950 & $0.52 \pm 0.10$ & 26.26 \\
J1503.7+6850 & $15: 04: 13.1$ & $+68: 56: 12$ & 0.32 & 4.622 & 1230 & $2.29 \pm 0.46$ & 26.82 \\
4C +63.22 & $15: 23: 45.8$ & $+63: 39: 24$ & 0.20 & 3.268 & 790 & $3.30 \pm 0.66$ & 26.53 \\
Mrk 1498 & $16: 28: 03.8$ & $+51: 46: 30$ & 0.05 & 0.965 & 1150 & $2.60 \pm 0.52$ & 25.16 \\
4C +34.47 & $17: 23: 20.6$ & $+34: 18: 00$ & 0.21 & 3.395 & 1120 & $5.21 \pm 1.04$ & 26.77 \\
\hline
\end{tabular}

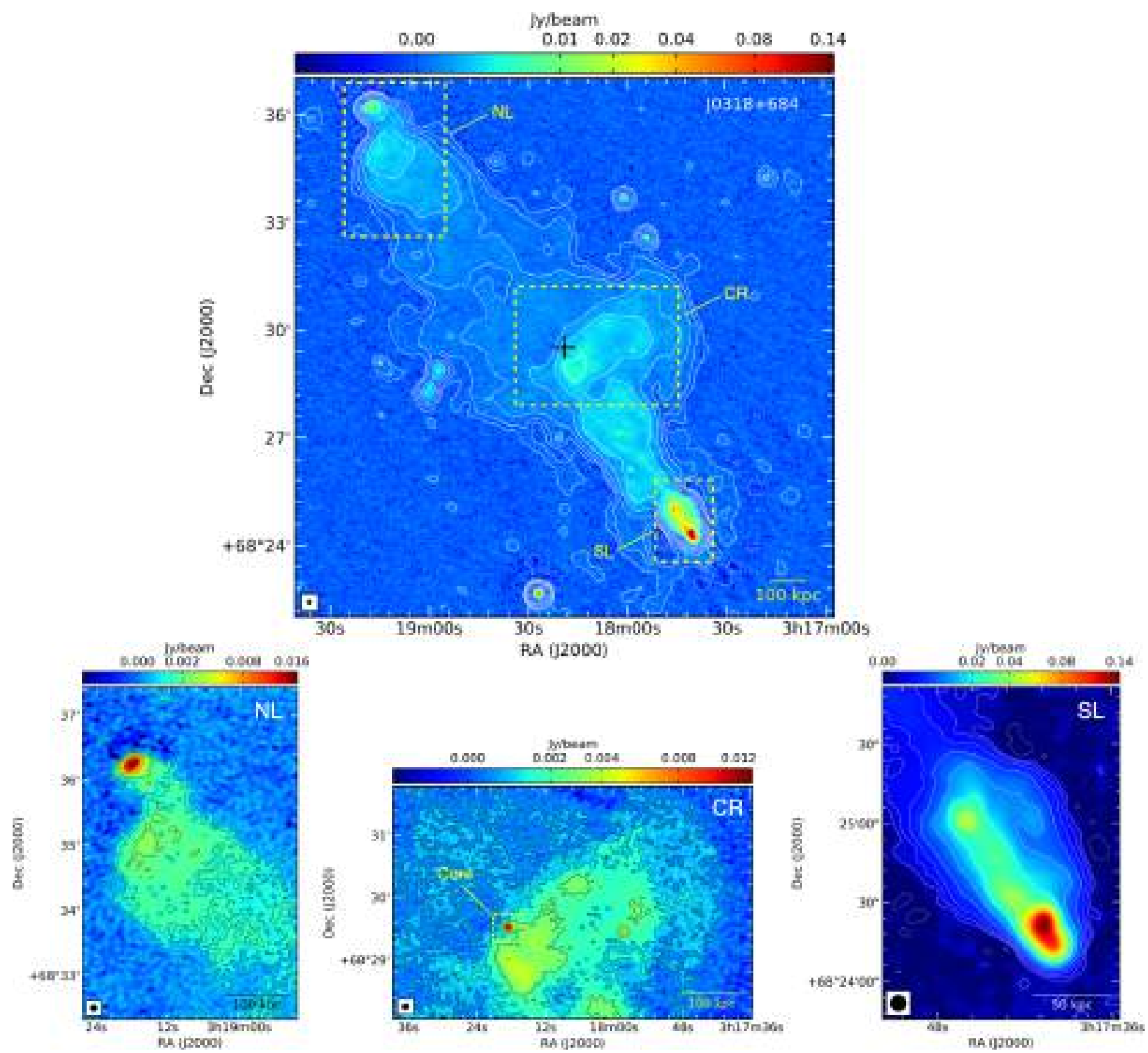

Figure 1. Top panel: LOFAR image of the source J0318+684. The colour scale represents the 6 arcsec resolution image, while contours are from the 20 arcsec one. Bottom panels: zoom on the northern lobe (left), core region (middle), and southern lobe (right) from the LOFAR image at 6 arcsec resolution. Contours are multiples of the image rms, namely $3 \times$ rms $\times(-1,1,2,4,8,16,32,64,128,256)$. Negative contours are dashed. The beam is shown in the lower left-hand corner. 

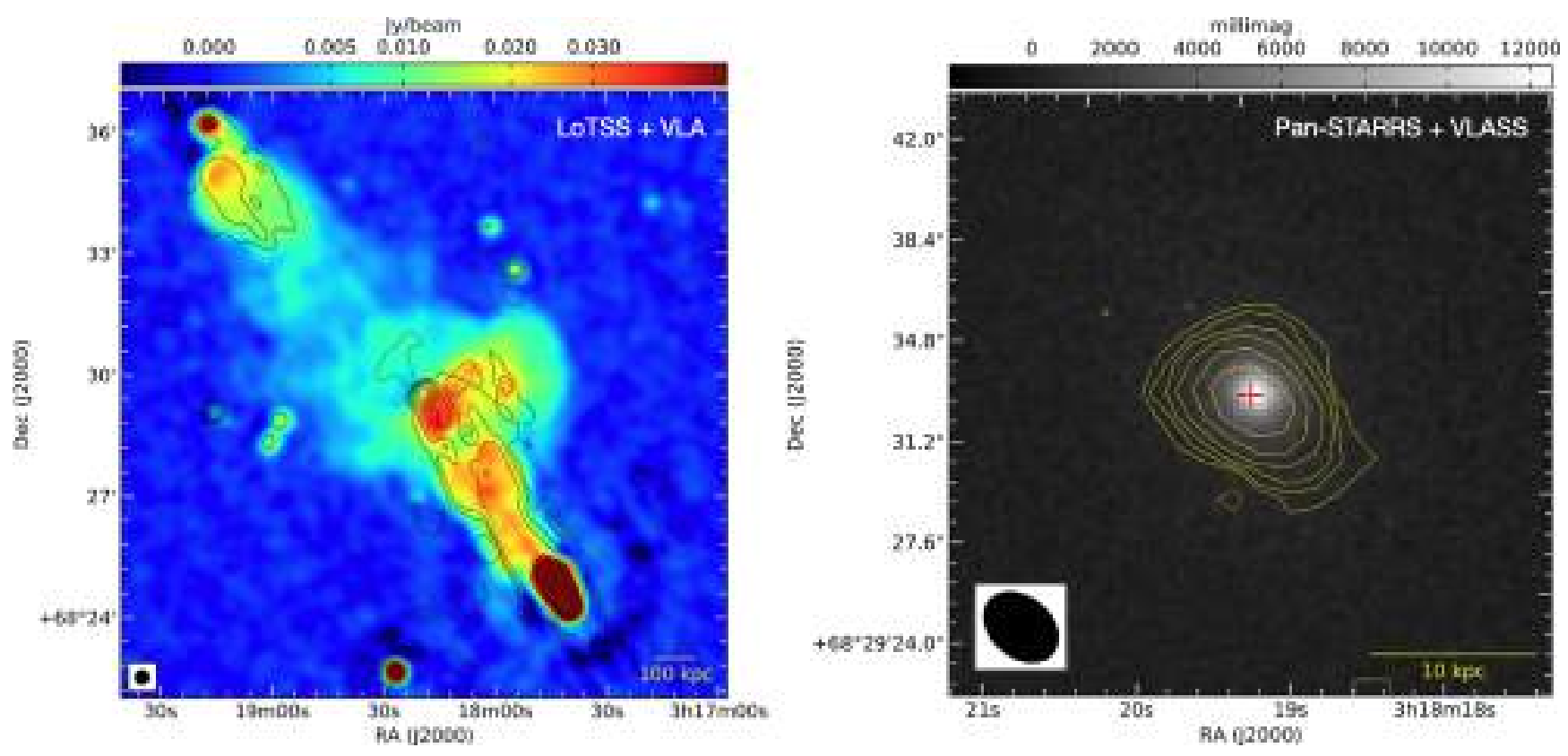

Figure 2. J0318+684. Left-hand panel: comparison between LOFAR (144 MHz, in colours) and VLA ( $3 \mathrm{GHz}$, in contours) images at a matched resolution of 20 arcsec. The beam is shown in the lower left-hand corner. Right-hand panel: Pan-STARRS $i$-band image of the host galaxy, with VLASS contours superimposed (2-4 GHz, $3 \times 2 \operatorname{arcsec}^{2}$ angular resolution). Contours are multiples of the image rms, namely $3 \times \mathrm{rms} \times(-1,1,2,4,8,16,32,64,128,256)$. Negative contours are dashed. The VLASS beam is shown in the lower left-hand corner.

can originate the deflection when they are tilted with respect to the jet axis. From a simple bidimensional Gaussian fit of the optical emission from the Pan-STARRS image, we estimated a PA of $31^{\circ} \pm 7^{\circ}$ for the host galaxy major axis, consistent with the one measured for the southern jet axis $\left(\sim 34^{\circ}\right)$ : this alignment between jet and host galaxy major axis does not support the mentioned backflow scenario. Alternatively, Gillone, Capetti \& Rossi (2016) showed how the secondary radio structure of $\mathrm{X}$-shaped radio galaxies is often oriented towards the minor axis of the host galaxy, proposing that it originates from the expansion of the radio cocoon in an asymmetric external medium. This scenario is compatible with the previously discussed orientation of the radio and optical counterparts of this source (based on the jet axis and host galaxy major axis orientation, respectively), suggesting that the diffuse emission perpendicular to the jet axis could have this origin.

\section{$3.2 \mathrm{~J} 0801.7+4764$}

The LOFAR image presented here (Fig. 3) shows a typical FRII morphology, with the northern lobe brighter than the southern one. The southern lobe presents a brighter region composed of three knots at $\sim 2.5$ arcmin from the core (marked as A in the figure). A check with Pan-STARRS images does not show correspondence with any host galaxy, suggesting that these are either real features along the jet, or radio emission from distant galaxies that faint emission is not detected in the optical band (see e.g. Baldi et al. 2013). The same is true for the bright-spot westwards of the northern hotspot (marked as $\mathrm{B}$ in the figure) that lies on a region of diffuse emission between the core and the jet. Both A and B component have a spectral index $\alpha_{0.14}^{1.44}=-0.91 \pm 0.06$, calculated between LOFAR and NVSS data, as expected for diffuse regions in the lobes. Kuźmicz \& Jamrozy (2012) provided an estimate of the jet inclination angle with respect to the line of sight, on the basis of the lobes flux density ratio, and assuming it is due to Doppler boosting: they obtained a value of $37^{\circ}$,

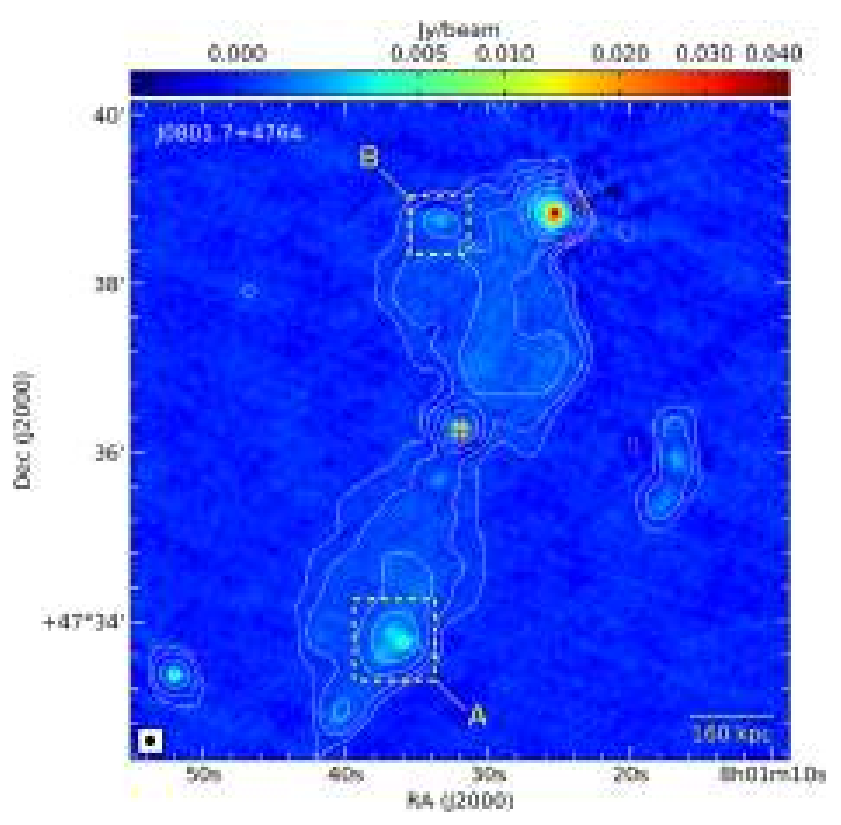

Figure 3. LOFAR image of the source J0801.7+4764. The colour scale represents the 6 arcsec resolution image, while contours are from the 20 arcsec one. Contours are multiples of the image rms, namely $3 \times$ rms $\times(-1,1,2,4$, $8,16,32,64,128,256)$. Negative contours are dashed. The cross indicates the $\mathrm{X}$-ray position. The 6 arcsec beam is shown in the bottom left-hand corner.

larger than the typical maximum angle of $\sim 10^{\circ}$ found for blazars, but still resulting in an asymmetry in jets brightness. Following their argument, the northern lobe is the one oriented towards the observer, while the southern lobe is the receding one. Information about jet axis inclination allows us to provide an improved estimate of the physical size of the source. The source size as measured from LOFAR observations, from lobe to lobe, is 440 arcsec, which 

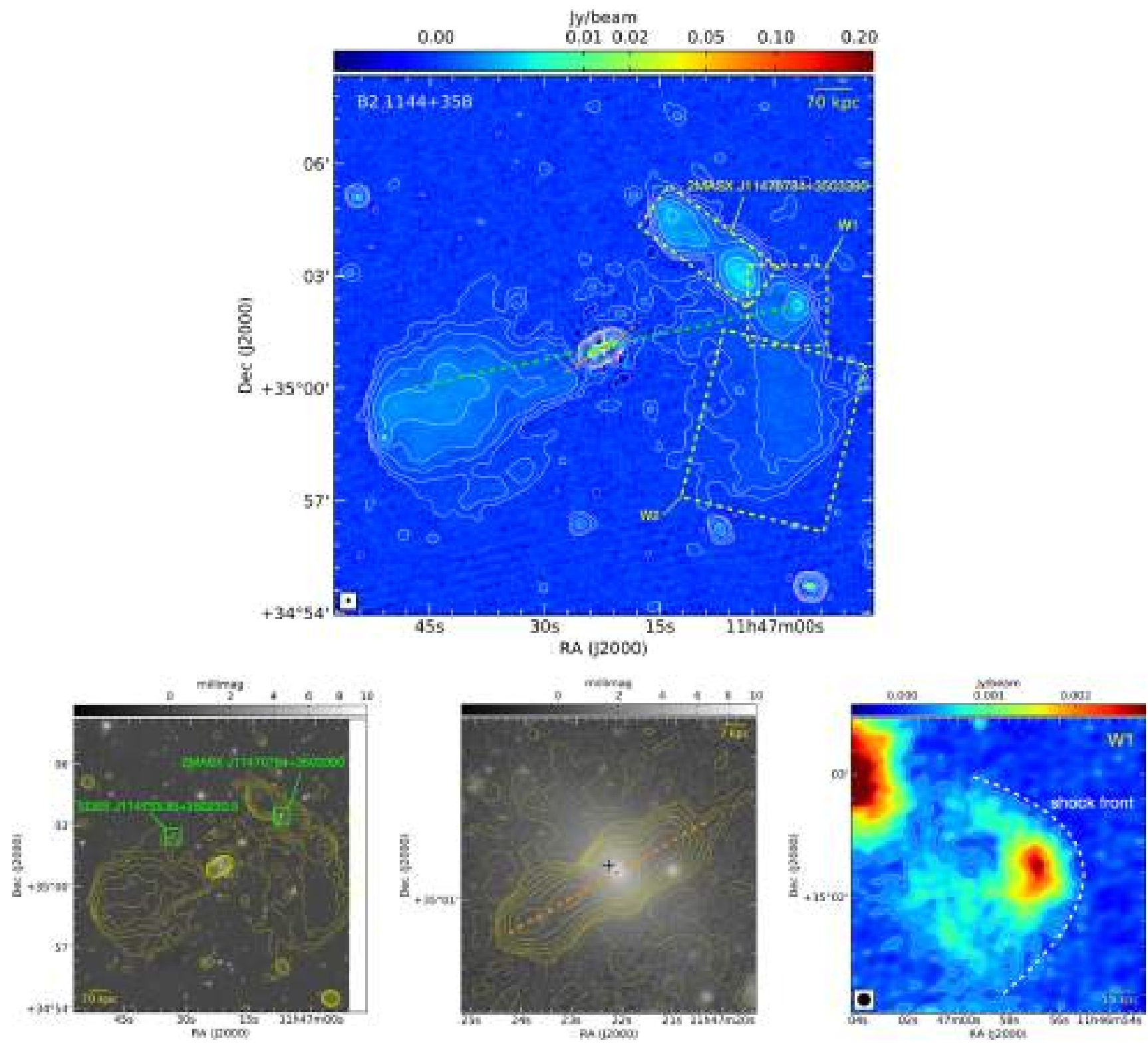

Figure 4. Top panel: LOFAR images of source B2 1144+35B. The colour scale represents the 6 arcsec resolution image, while contours are from the 20 arcsec one. The jet axis connecting the core to component W2 is indicated as a red dashed line, while the axis of the inner jet as an orange dashed line. The LOFAR 6 arcsec beam is shown on the lower left-hand corner. The cross indicates the X-ray position. Bottom left-hand panel: overlay of the LOFAR 20 arcsec contours with the Pan-STARRS $i$-band image. The position of the nearby galaxy 2MASX J11470784+3503390 is indicated as a red box. Bottom middle and bottom right-hand panels: zoom on the core region (LOFAR 6 arcsec contours overlaid to Pan-STARRS $i$-band image) and on the W1 component (both colours and contours from LOFAR 6 arcsec image), respectively. The LOFAR 6 arcsec beam is shown on the lower left-hand corner. For all images contours are multiples of the image rms, namely $3 \times$ rms $\times(-1,1,2,4,8,16,32,64,128)$. Negative contours are dashed.

translates into a projected linear size of $1.2 \mathrm{Mpc}$ at the redshift of the source. Considering an inclination angle of $37^{\circ}$ for the jet axis, the deprojected linear size of the source is $2 \mathrm{Mpc}$. In Appendix B, we report the serendipitous discovery of a radio galaxy in the LoTSS field of this GRG.

\subsection{B2 1144+35B}

Giovannini et al. (1999) and Giovannini, Giroletti \& Taylor (2007) performed a detailed study of this source through multiscale observations, from pc to kpc. In particular, they found discontinuities in the jet morphology through VLA observations, suggesting an intermittent activity. The LOFAR image at 20 arcsec resolution (Fig. 4, top panel) highlights diffuse, extended emission around the lobes, and, in particular, southwards of the western one (indicated as W2 in Fig. 4). The latter was partially reconstructed by the VLA image at $1.4 \mathrm{GHz}$ in Giovannini et al. (1999), and tentatively identified as the Western lobe. The LOFAR image presented here recovers a larger emission region, both in north-south (NS) and east-west (EW) directions, at a frequency a factor of 10 lower. We could estimate the spectral index of the W2 region using LOFAR and NVSS data, although in the latter only the brightest part is detected (see Bruni et al. 2020): we obtained a value of $\alpha_{0.14}^{1.44}=-0.92 \pm 0.06$. This is consistent with the value estimated by Giovannini et al. (1999) 
between 1.4 and $5 \mathrm{GHz}$, of about -1 . In the NW sector a radio galaxy is present (corresponding to object 2MASX J11470784+3503390), moreover at the same redshift as our target $(z=0.06)$, and partially overlapping with its line of sight. Indeed, the host galaxy of our target matches with one of the clusters from the Smith et al. (2012) catalogue (identified with ID 2259 in that work, see Section 4). A further galaxy, named SDSS J114733.85+350230.5, also at the same redshift as our GRG, is located north-east of the core and is partially overlapping with the Eastern lobe (see Fig. 4, bottom left-hand panel).

Despite the complexity of the field, the Western lobe of our GRG is well recognizable thanks to the bow shock visible around the hotspot (indicated as W1 in Fig. 4, top panel), meaning that the jet is expanding in a dense environment, and allowing to draw the jet axis back to the core. A zoom on feature W1 is presented in Fig. 4 (bottom right-hand panel). Similarly to what done for $\mathrm{W} 2$, we estimated a spectral index of $\alpha_{0.14}^{1.44}=-0.73 \pm 0.06$ for this component, consistent with the value of about -0.8 estimated by Giovannini et al. (1999) between 1.4 and $5 \mathrm{GHz}$. The inner region of the source $(\sim 50$ arcsec, corresponding to a projected linear size of $\sim 60 \mathrm{kpc}$ ) is resolved in the 6 arcsec resolution image (see Fig. 4, bottom-centre panel), showing a structure with multiple components: a core (the brightest one), coincident with the optical position of the host galaxy, as well as with the X-ray counterpart of the AGN (Bruni et al. 2020), and three knots along the jets. The axis connecting these components has a PA of $\sim-60^{\circ}$, while the one traced between the core and the Western lobe (W1) has a PA of $\sim-77^{\circ}$ (see top panel of Fig. 4). With a misalignment between the two of $\sim 17^{\circ}$, a counter-clock reorientation of the jet axis on a Myr time-scale can be supposed. Interestingly, an S-shaped morphology of the Eastern jet was shown by the VLA observations at $4.9 \mathrm{GHz}$ presented in Giovannini et al. (1999): this is typically expected from jet precession (see Krause et al. 2019; Horton, Krause \& Hardcastle 2020 and references therein) and would support the reorientation discussed above. Nevertheless, none of these two axes seem to be aligned with the hotspot visible in the Eastern lobe.

We can compare this source with a similar case of GRG reported by Subrahmanyan et al. (2006), and termed fat-double due to the noticeable transverse extension of the lobes with respect to the lobecore distance (SGRS J0515-8100). Those authors suggested that the presence of distorted lobes could originate from an intermittent jet activity, with multiple axis changes, due to gravitational perturbations from a companion galaxy. Indeed, the optical counterpart of our target presents a boxy shape, as pointed out by previous authors (Giovannini et al. 1999), possible aftermath of a merger event. Moreover, the correspondence with a galaxy cluster mentioned above suggests that interactions within the cluster members could be ongoing, making the jet evolution more complex than the case of an isolated radio galaxy. Another example of source with a morphology comparable with this GRG was presented by Liu et al. (2019). Those authors, through an analysis of X-ray cavities and radio jet axis in $3 \mathrm{C} 88$, concluded that a jet reorientation during the last $\sim 10^{7} \mathrm{yr}$ is the most likely explanation for the observed radio structure. As a whole, the LOFAR images presented here are consistent with a fat-double morphology, suggesting either multiple radio phases with different jet axes, or a jet reorientation on a Myr time-scale.

\section{$3.4 \mathrm{~J} 1153.9+5848$}

From the LOFAR 20 arcsec resolution contours of this source (Fig. 5, top left-hand panel) two symmetric tails of faint emission are detected for the first time, towards both the north and south sides with respect to the core, and with an extension larger than the one of the ongoing radio phase. The contours highlight the presence of bright-spots at the end of the tails (named $\mathrm{H} 1$ and $\mathrm{H} 2$ in Fig. 5, top left-hand panel). The axis connecting the peaks at the edges of the structure intercepts the core region, and has a PA of $\sim-4^{\circ}$. As a comparison, the axis connecting the main lobes of the source shows a PA of $\sim+36^{\circ}$. Both axes are shown as dashed lines in Fig. 5. The fact that the distance between these two features is larger than the one between the lobes, allows us to reasonably exclude a backflow origin (see a similar case in Dennett-Thorpe et al. 2002). These tails were not previously detected in NVSS (see Bassani et al. 2021). However, that survey is shallower than the LOFAR observations presented here (the typical rms level of NVSS is $\sim 0.5 \mathrm{mJy}_{\text {beam }}{ }^{-1}$, four times larger than the one in our LOFAR 20 arcsec resolution image), and with a poorer short-baselines coverage (thus a lower sensitivity to extended structures). Moreover, the expected peak flux density at the NVSS frequency $(1.4 \mathrm{GHz})$, assuming a typical spectral index $\alpha=$ -1.5 for relic plasma, would be a factor of 30 lower than the one in our LOFAR images (decreasing from $\sim 1.5$ to $\sim 0.05 \mathrm{mJy}_{\text {beam }}^{-1}$ ), laying an order of magnitude below the NVSS noise level. We could estimate an upper limit of $\alpha_{0.14}^{1.44}<-0.52$ and $\alpha_{0.14}^{1.44}<-0.75$ for $\mathrm{H} 1$ and $\mathrm{H} 2$, respectively, given an rms noise of $0.5 \mathrm{mJy}^{\text {beam }}{ }^{-1}$ in the corresponding regions of NVSS images. Further tailored observations, covering the $\mathrm{MHz}$ to $\mathrm{GHz}$ frequency range, could help in determining the age of these structures.

In case the hypothesis of a remnant radio structure is confirmed, this could be a genuine case of $\mathrm{X}$-shaped radio galaxy, with a change in the radio axis orientation of almost $40^{\circ}$. This class of sources is among the best candidate to host a binary supermassive black holes (BSBHs) system (Saripalli \& Roberts 2018). Such a link between empirical evidences of bending jets and the possibility of a geodetic precession in a binary system of AGN was already proposed decades ago (Begelman, Blandford \& Rees 1980). Recently, 3D hydrodynamic simulations of kpc-scale radio galaxies with a precessing jet (Horton et al. 2020) allowed to test the formation of the peculiar morphologies among these objects (e.g. X-shaped and S-shaped; see Krause et al. 2019 for a summary). Following the work of those authors, the analogous case of J1153.9+5848 could be attributable to a jet with a precessing angle of $\sim 45^{\circ}$ that would form an X-shaped morphology on a Myr time-scale with two axes of comparable length. Those authors explain such a jet precession in terms of a BSBHs system, formed during a merger with another galaxy. However, alternative explanations have been proposed in the literature, including jet axis realignment (Natarajan \& Pringle 1998), and a precessing accretion disc (Kurosawa \& Proga 2008). Highresolution (pc-scale) radio observations could help to disentangle the possible scenarios for this source: in case two radio-emitting black holes are present in its core, they could be detected as flat-spectrum components, analogously to the case of NGC 7674 (Kharb, Lal \& Merritt 2017).

\section{$3.5 \mathrm{J1238.4+5349}$}

This source was included in the GRG catalogue compiled from the first data release of LoTSS (Dabhade et al. 2020a). The full resolution image (Fig. 5, bottom left-hand image) shows two lobes in EW direction and two compact components in the middle. On the one hand, the easternmost among the latter ones corresponds to the $\mathrm{X}$ ray position from our previous works, and shows a counterpart in the Pan-STARRS $i$-band image, and is thus coincident with the host galaxy of our GRG (see Fig. 5, bottom right-hand panel). On the other hand, the western component of the inner couple does not correspond to any known source in the SIMBAD astronomical data 

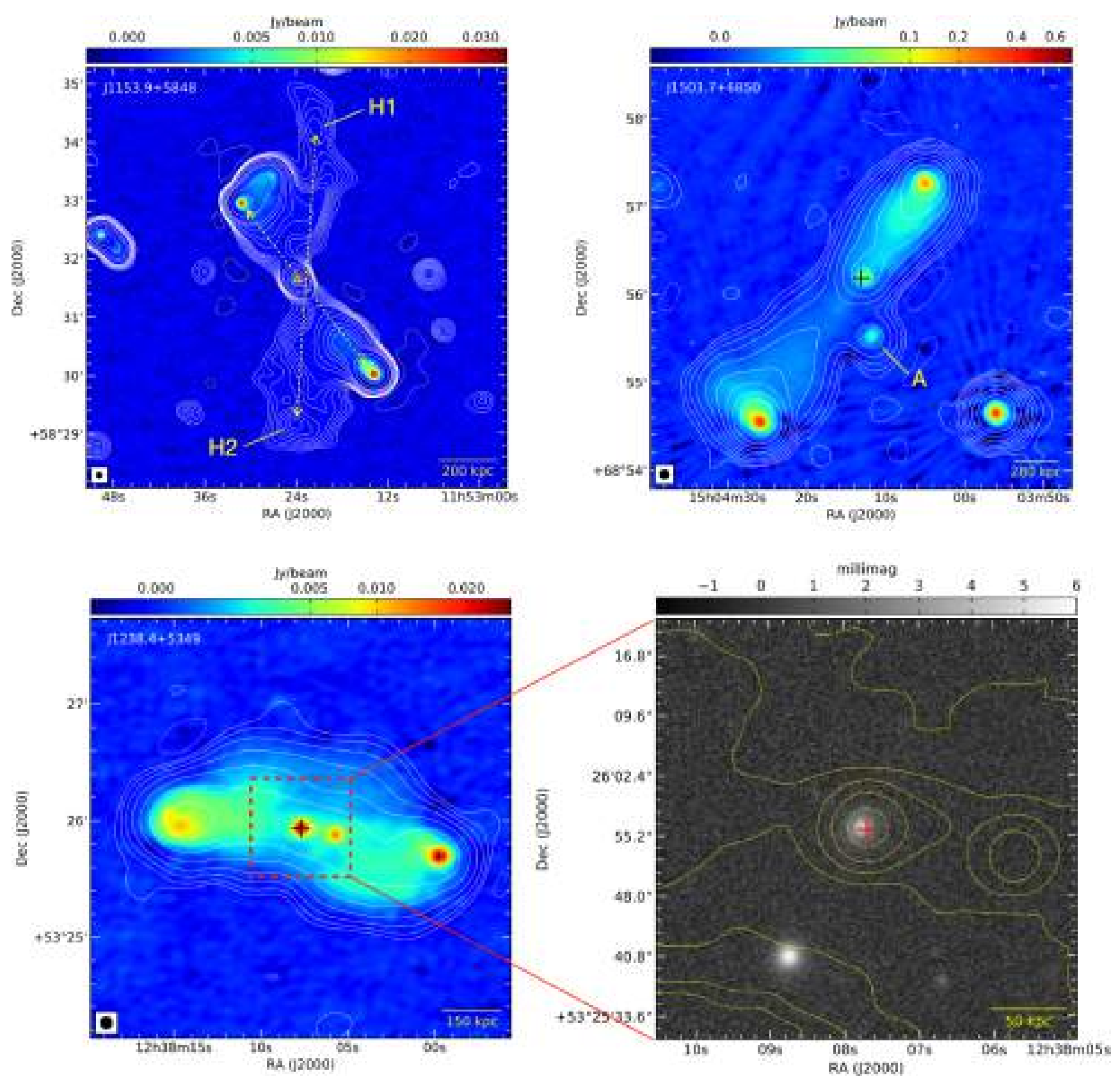

Figure 5. LOFAR images of sources J1153.9+5848, J1503.7+6850, and J1238.4+5349. The colour scale represents the 6 arcsec resolution image, while contours are from the 20 arcsec one. Contours for J1153.9+5848 are the image rms $\times(-3,3,4,5,6,7,8,9,10,15,20,30,40,50,100,200,300,400,500)$, while for sources J1503.7+6850 and J1238.4+5349 are $3 \times \mathrm{rms} \times(-1,1,2,4,8,16,32,64,128,256)$. Negative contours are dashed. The 6 arcsec beam is shown in the lower left-hand corner. Bottom right-hand panel: zoom on the core region of J1238.4+5349, with LOFAR contours at 6 arcsec resolution overlaid to the Pan-STARRS $i$-band image. The cross indicates the $\mathrm{X}$-ray position.

base (Wenger et al. 2000), and could thus be interpreted as a knot along the jet. Finally, in the 6 arcsec resolution image from LOFAR a curved, symmetric emission is visible between the lobes and the core (from the eastern lobe towards north, and from the western lobe towards south), resembling the typical morphology of a backflow.

\subsection{J1503.7+6850 $(4 \mathrm{C}+69.18)$}

An asymmetric emission is visible for this source (Fig. 5, top righthand panel), with the northern one being brighter than the southern one. Kuźmicz \& Jamrozy (2012) discussed how this source almost lies on the plane of the sky, with an inclination angle of $81^{\circ}$ with respect to line of sight. This could suggest that the difference in jets brightness could be due to a denser medium probed by the northern jet (see source Mrk 1498 below for a similar case). An additional emission spot (indicated as A in the figure) is visible southward of the core: it corresponds to an unidentified optical source in Pan-STARRS, and should thus not be considered as part of the GRG emission. At the lower resolution of NVSS it is not resolved from the core. Lara et al. (2001) presented a VLA image of this source at $1.4 \mathrm{GHz}$, and 10 arcsec resolution: the same morphological properties are visible, including component A that shows a peak flux density of about 6 

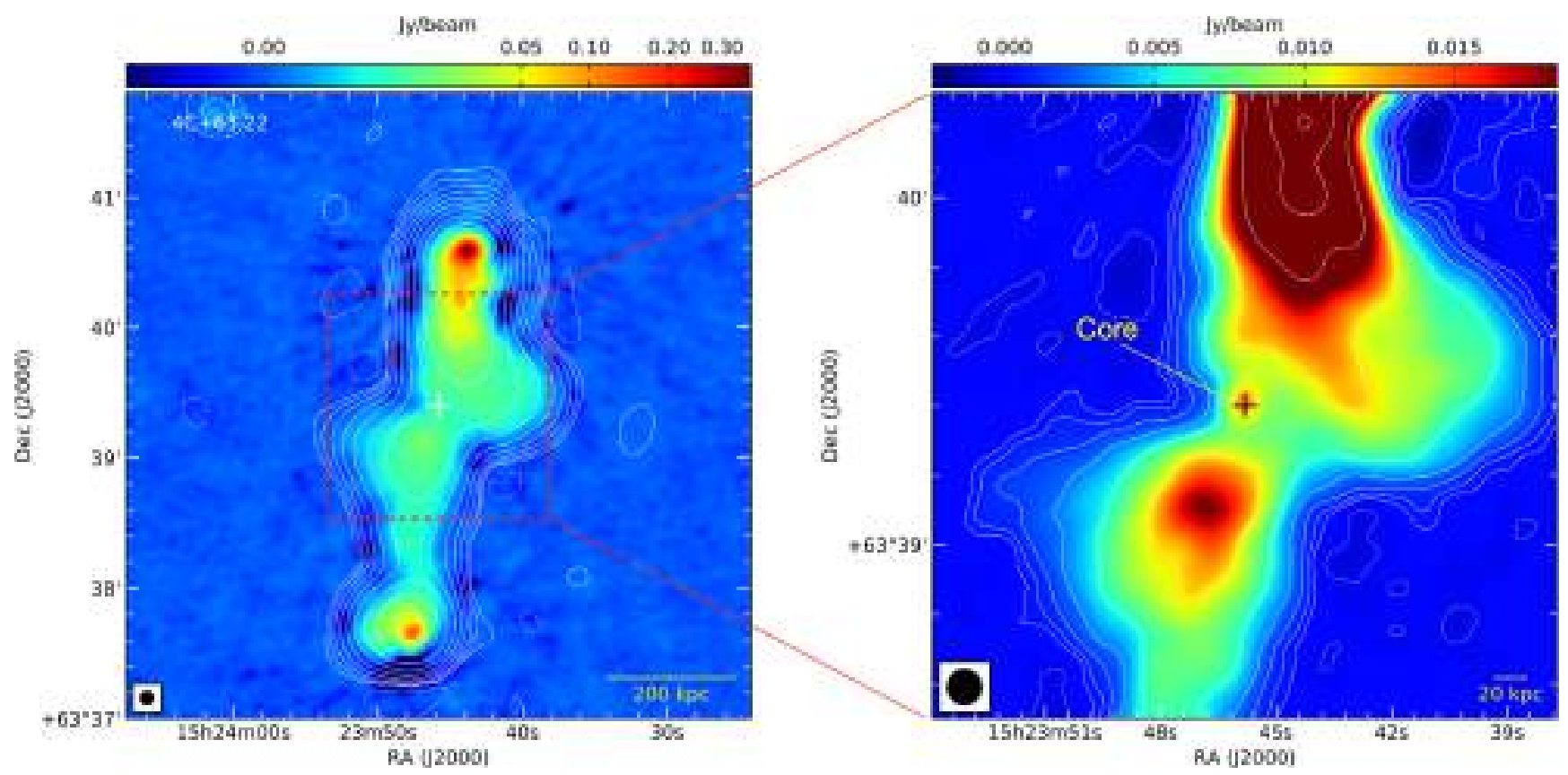

Figure 6. LOFAR images of source $4 \mathrm{C}+63.22$. Left-hand panel: the colour scale represents the 6 arcsec resolution image, while contours are from the 20 arcsec one. Right-hand panel: zoom on the core region, both colour scale and contours are drawn from the 6 arcsec resolution image. Contours are multiples of the image rms, namely $3 \times \mathrm{rms} \times(-1,1,2,4,8,16,32,64,128,256)$. Negative contours are dashed. The cross indicates the X-ray position. The 6 arcsec beam is shown in the lower left-hand corner.

mJy beam ${ }^{-1}$, resulting in a spectral index $\alpha_{0.14}^{1.44} \sim-0.45$. No signs of restarted radio activity are evident in the LOFAR image or previous ones from the literature, showing a typical FRII morphology.

\section{$3.74 \mathrm{C}+63.22$}

This source was presented as a tentative $\mathrm{X}$-shaped radio galaxy in Bruni et al. (2020), based on the TIFR GMRT Sky Survey (TGSS; Intema et al. 2017) image at $150 \mathrm{MHz}$, which shows hints of emission distributed in almost perpendicular direction with respect to the main jet axis. The AGN X-ray position nicely corresponds with the radio core. The nature of the diffuse off-axis emission between the lobes and the core was not clearly established at the resolution of the available image ( 25 arcsec). The LOFAR image presented here (Fig. 6) offers an improvement in angular resolution by a factor of $\sim 4$, and a factor of $\sim 10$ in sensitivity, confirming the $X$-shaped morphology. None the less, we cannot easily classify the off-axis emission as either remnant lobes from a previous radio phase, or plasma backflow created by the present jets. Indeed, the southern region looks like plasma expanding in the direction from the core region towards to external medium, while the northern one presents the typical backflow morphology, with a broadening from the lobe towards the core. Finally, the optical images available from surveys are not of sufficient resolution to measure the major axis orientation, and test the scenario proposed by Gillone et al. (2016) previously invoked for source J0318+684. Further observations, aiming at studying the plasma aging across these structures, will allow us to shed light on the evolution of this source.

\subsection{Mrk 1498}

This source was the subject of a multiwavelength study by Hernández-García et al. (2019), who discussed a possible reactivation of the nuclear activity following a merger event. Indeed, the source presents an obscured AGN, with a GPS in its core region (Bruni et al. 2019). Bruni et al. (2020) discussed the morphological properties visible in the TGSS map at $150 \mathrm{MHz}$ (25 arcsec resolution), where basically the core and lobes were detected, while the jet connecting them was not present. The LOFAR image (Fig. 7, top panel) shows for the first time details about the complex structure along the jet, with a bent northern jet, and a branched southern lobe, with several knots (see a zoom on this region in Fig. 7, bottom right-hand panel). A hint of the two southern jet branches was already visible in the TGSS image, although the lower resolution did not allow to fully distinguish them. The overall source morphology is asymmetric, with the northern structure having almost twice the extension of the southern one (11 arcmin versus 7.5 arcmin, from core to hotspot). A similar asymmetry was discussed by Shulevski et al. (2019) for $3 \mathrm{C} 236$, explaining it as a difference in the IGM probed by the two jets. This hypothesis may hold also for Mrk 1498, moreover it could explain the enhanced surface brightness and forked morphology of the southern jet and lobe, as a result of a strong interaction with a dense medium.

Thanks to the enhanced sensitivity of these LOFAR images, it is possible to highlight an emission gap between the well-defined core and the inner edge of the southern jet structure (see Fig. 7, bottom lefthand panel). A similar gap is visible between the core and the edge of northern lobe, although the latter is less defined if compared with the previous one. On the southern side, we can measure a separation of about 40 arcsec (corresponding to $38 \mathrm{kpc}$ at the redshift of the source) between the core and the first edge of southern emission region. A similar gap is visible in archival VLA observations at $5 \mathrm{GHz}$ (35 $\times 15 \operatorname{arcsec}^{2}$ resolution, see Fig. 7, bottom left-hand panel). This is likely due to a collimated jet that in the region near to the core emits most of its radiation in the propagation direction, and with small energetic losses due to interaction with the medium, making it almost undetectable when lying on the plane of the sky. However, the presence of a GPS in the core of this GRG - as also confirmed by 

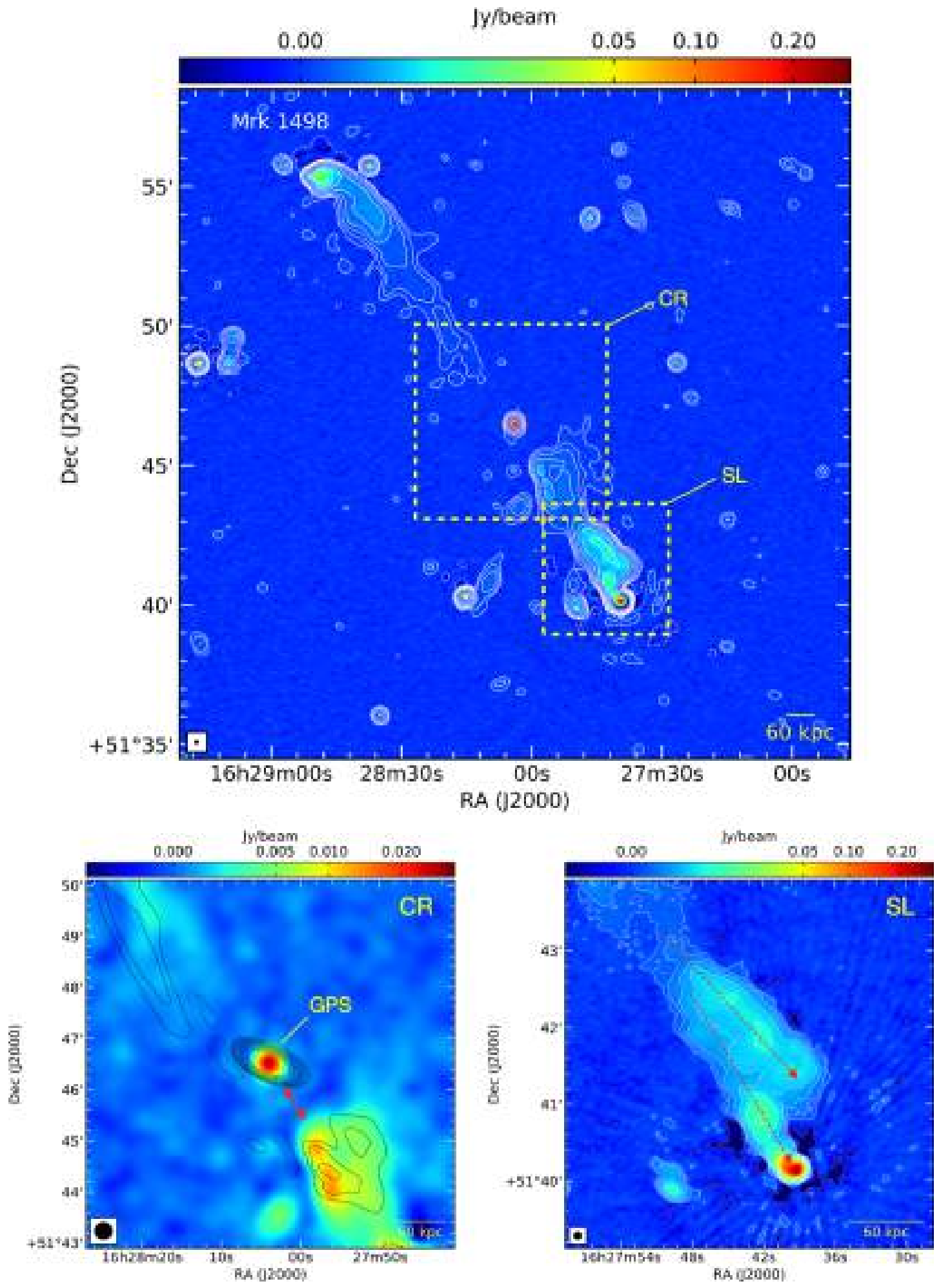

Figure 7. LOFAR images of source Mrk 1498. Top panel: the colour scale represents the 6 arcsec resolution image, while contours are from the 20 arcsec one. The 6 arcsec beam is shown in the lower left-hand corner. The cross indicates the X-ray position. Bottom left-hand panel: overplot of the VLA contours at $5 \mathrm{GHz}$ from archival observations ( $35 \times 15 \operatorname{arcsec}^{2}$ resolution) on the LOFAR $20 \operatorname{arcsec}$ resolution image. The emission gap of $\sim 40 \mathrm{kpc}$ between the core and the edge of the southern emission region, visible with both instruments, is marked in red. The 20 arcsec LOFAR beam is shown in the lower left-hand corner. Bottom right-hand panel: zoom of the LOFAR image at 6 arcsec resolution on the southwestern lobe, with the two branches marked with a red dashed line. The 6 arcsec beam is shown in the lower left-hand corner. For all images, contours are multiples of the image rms, namely $3 \times$ rms $\times(-1,1,2,4,8,16,32,64$, 128). Negative contours are dashed. 

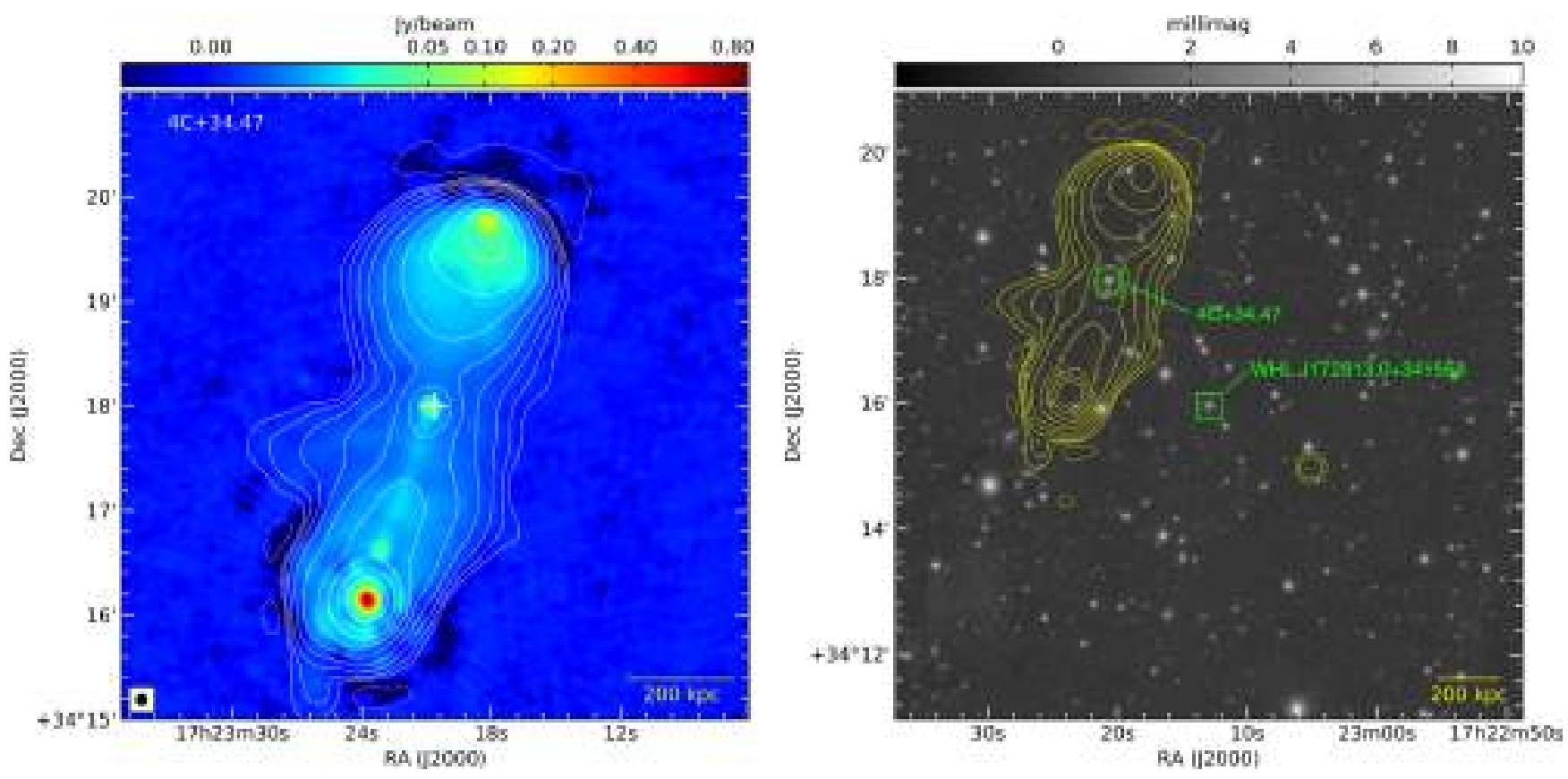

Figure 8. Left-hand panel: LOFAR image of sources $4 \mathrm{C}+34.47$. The colour scale represents the 6 arcsec resolution image, while contours are from the 20 arcsec one. The LOFAR 6 arcsec beam is shown in the lower left-hand corner. The cross indicates the X-ray position. Right-hand panel: overlay of the LOFAR 20 arcsec resolution contours with the Pan-STARRS $i$-band image centred on the brightest cluster galaxy WHL J172313.0+341558. Contours are multiples of the image rms, namely $3 \times \mathrm{rms} \times(-1,1,2,4,8,16,32,64,128,256)$. Negative contours are dashed.

the symmetric morphology visible from archival radio images at pcscale resolution (Hernández-García et al. 2019) - seems to suggest that the radio emission in the core region could be from a phase disconnected from the one responsible of the Mpc-scale structure.

\section{$3.94 \mathrm{C}+34.47$}

This source presents a well-defined core in the LOFAR image (Fig. 8, left-hand panel), previously classified as a GPS (Bruni et al. 2019). Both southern and northern lobes have bright and well-defined hotspots, sign of an active fuelling from the jets. The northern jet is not visible, probably due to a Doppler dimming effect, while the southern jet shows a knotty structure between the core and the lobe. These properties were not revealed at the lower resolution of the TGSS image discussed in Bruni et al. (2020). Hocuk \& Barthel (2010) first revealed the knotty structure of the southern jet from VLA observations at $1.4 \mathrm{GHz}$ and $5 \mathrm{GHz}$, and also provided an estimate of the jet angle to line of sight. On the basis of the jet/counter-jet flux density ratio, and the jet bulk velocity, they infer a jet axis angle within $57^{\circ}$ from the line of sight. A knotty, discontinuous, jet could be the result of an intermittent activity of the radio core. Another option could be an alternating recollimation/expansion of the jet, resulting in an enhanced flux for the collimated components. Alternatively, a helical jet could produce a similar morphology, with brighter knots corresponding to regions where the jet orientation is close to the line of sight. In Appendix A, we present an archival Chandra image in the $0.5-7 \mathrm{keV}$ energy range, were the southern hotspot and a knot are detected.

Finally, we notice that this GRG can be associated with the galaxy cluster WHL J172313.0+341558 from the Wen, Han \& Liu (2012) catalogue (see Section 4). Fig. 8 (right-hand panel) shows the position of the GRG relative to the brightest cluster galaxy (BCG), having a $z=0.1955$.

\section{ENVIRONMENT ANALYSIS}

In order to evaluate the influence of environment on the morphology of these GRGs, we adopted the same strategy of recent works from the LoTSS Collaboration (Croston et al. 2019; Dabhade et al. 2020a) to investigate the presence of galaxy clusters potentially associated with these GRGs. In particular, we considered the following galaxy clusters catalogues: the Gaussian Mixture Brightest Cluster Galaxy (GMBCG) catalogue (Hao et al. 2010), including 55880 clusters from the Sloan Digital Sky Survey (SDSS) Data Release 7 (DR7) in the redshift range $0.1<z<0.55$; the Wen et al. (2012) catalogue, consisting of 132684 clusters drawn from SDSS Data Release 8 (DR8) in the redshift range $0.05<z<0.8$; and the red-sequence matched-filter Probabilistic Percolation (redMaPPer) catalogue (Rykoff et al. 2014), also based on SDSS DR8, and providing more than 25000 clusters in the redshift range $0.08<$ $z<0.55$. In addition to those works, we considered the Smith et al. (2012) catalogue, presenting 10443 groups and clusters from SDSS DR7.

Six out of nine GRGs studied here, while nine among the 23 constituting the whole HXGRGs sample, are included in the footprint of the mentioned works, not allowing statistical considerations on the full sample. Analogously to Croston et al. (2019), we considered as criteria for the clusters matching a physical distance $\Delta D \leq 1$ $\mathrm{Mpc}$ at the redshift of our objects, and a maximum difference in redshift $\Delta z \leq 0.01$. In this way, we found an association for two sources out of six: B2 1144+35B, matching with one of the clusters from the Smith et al. (2012) catalogue (identified with ID 2259 in that work), and $4 \mathrm{C}+34.47$, associated with the galaxy cluster WHL J172313.0+341558 from the Wen et al. (2012) catalogue. Both sources are included in the subsample of nine GRGs with LoTSS data presented here. The remaining four sources presented here that do not show a match with galaxy clusters are Mrk 1498, J0801.7+4764, $\mathrm{J} 1153.9+5848$, and $\mathrm{J} 1238.4+5349$. 


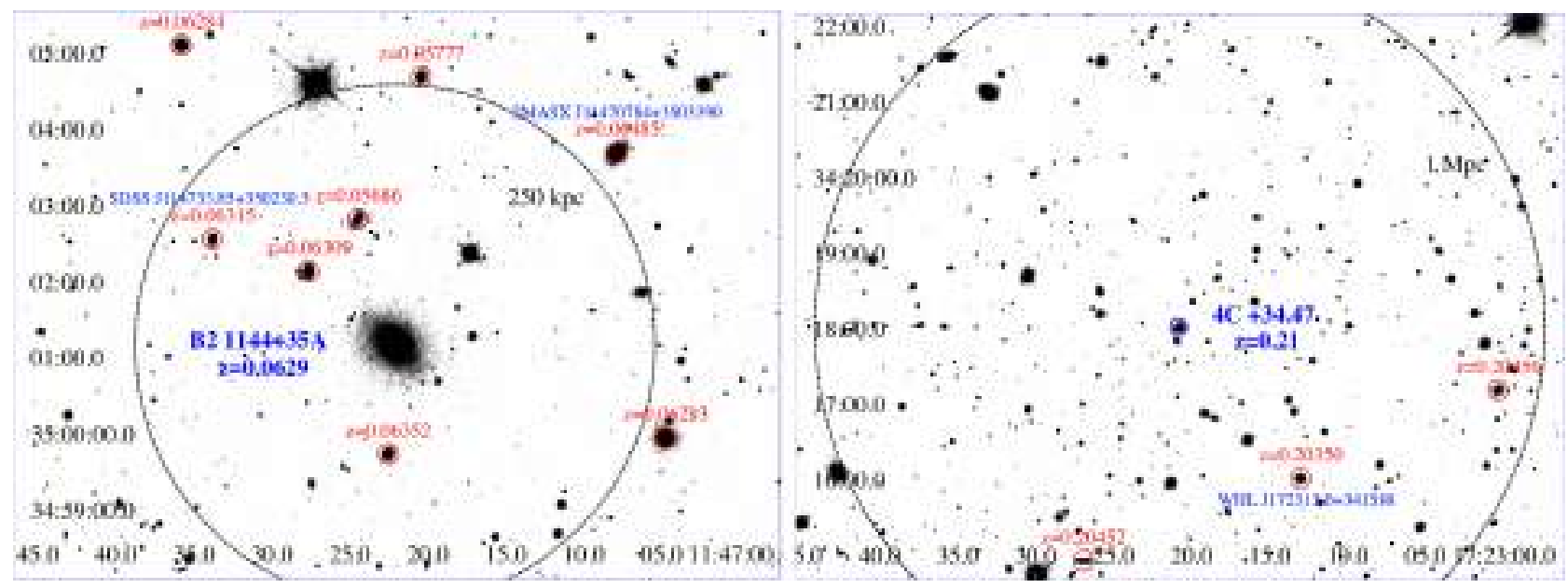

Figure 9. Left-hand panel: the SDSS $R$-band image of the inner field around B2 1144+35B (Ahn et al. 2012). The blue circle marks the position of the central radio galaxy while the larger dashed black circle corresponds to a distance of $250 \mathrm{kpc}$ computed at its redshift. Red circles mark the location of cosmological neighbours, i.e. optical galaxies having a redshift difference, with respect to that of the central radio galaxy, less than 0.005 (see Massaro et al. 2019 , $2020 \mathrm{~b}$ for more details), as well as that of SDSS J114720.45+350438.5 at $z=0.05777$ lying at $\sim 250 \mathrm{kpc}$ and SDSS J114724.51+350246.5 with a redshift estimate of 0.05686 at $\sim 120 \mathrm{kpc}$ distance, which could belong to the same cosmological structure. B2 1144+35B appears to be the BCG of a galaxy group/cluster. Finally, we highlight that the closest companion galaxy, SDSS J114722.74+350108.8 at $z=0.06272$, located at only $\sim 7 \mathrm{kpc}$ projected distance is not marked here. Right-hand panel: the SDSS $R$-band image of the field around 4C +34.47 (Ahn et al. 2012). The blue circle marks the position of the host elliptical galaxy of $4 \mathrm{C}+34.47$ while the larger dashed black circle corresponds to a distance of $1 \mathrm{Mpc}$ computed at the same redshift of the central radio galaxy (i.e. $z=0.21$ ) and centred on its position. Finally, red circles mark the location of three galaxies, lying within $1 \mathrm{Mpc}$, and having a redshift difference, with respect to that of the central radio galaxy, less than 0.005 (known as cosmological neighbours; see Massaro et al. 2019, 2020b). Their presence clearly indicate that 4C +34.47 belongs to a galaxy group/cluster.

To confirm this result, we performed an environment analysis adopting the same methods and procedures outlined in Massaro et al. (2019, 2020a,b). We searched for cosmological neighbours in the SDSS lying within the $2 \mathrm{Mpc}$ radius computed at the same redshift of the central radio galaxy, with all SDSS magnitude flags indicating a galaxy-type object, and having a maximum spectroscopic redshift difference $\Delta z \leq 0.005$ with respect to that of the central target, corresponding to the maximum velocity dispersion in groups and clusters of galaxies (see e.g. Moore, Frenk \& White 1993; Eke et al. 2004; Berlind et al. 2006). We could confirm a cosmological neighbours overdensities around B2 $1144+35 \mathrm{~B}$ and $4 \mathrm{C}+34.47$, in agreement with the results from the cross-match of the mentioned catalogues.

In the case of B2 $1144+35 \mathrm{~B}$, we found nine cosmological neighbours in addition to SDSS J114720.45+350438.5 at $z=$ 0.05777 lying at $\sim 250 \mathrm{kpc}$ and SDSS J114724.51+350246.5 with a redshift estimate of 0.05686 at $\sim 120 \mathrm{kpc}$ distance (see Fig. 9, left-hand panel). In particular three cosmological neighbours are located within a circular region of $250 \mathrm{kpc}$, one of them showing a prominent $\mathrm{H} \alpha$ in its optical spectra: SDSS J114733.85+350230.5, at $z=0.06315$ as occurs for SDSS J114702.75+350720.4 $(z=$ $0.06298)$ at larger distance. A very close companion galaxy, namely: SDSS J114722.74+350108.8 at $z=0.06272$ is also located at only $\sim 7 \mathrm{kpc}$ projected distance (this is not highlighted in Fig. 9). The probability to have this number of cosmological neighbours at $z$ $\sim 0.06$ is less than a few per cent, as shown in Massaro et al. (2019), thus strongly suggesting the presence of a galaxy cluster. Finally, we highlight that B2 $1144+35 \mathrm{~B}$ is most probably the BGC of this galaxy cluster being located at $\sim 70 \mathrm{kpc}$ from the centroid of all positions of all other cosmological neighbours. The distribution of the central region (i.e. within $250 \mathrm{kpc}$ ) of all SDSS optical sources, marked with their redshift estimate if present, is shown in Fig. 9.
For $4 \mathrm{C}+34.47$, we found three cosmological neighbours within $1 \mathrm{Mpc}$ distance from the position of the central radio galaxy, all marked with red circles in Fig. 9, right-hand panel. A fourth galaxy at $z=0.20479$ lies at $1.12 \mathrm{Mpc}$ in the north-western direction, being probably part of the same cosmological structure. With respect to B2 $1144+35 B, 4 C+34.47$ lies at higher redshift and given the number of cosmological neighbours we can certainly claim that also this radio galaxy is harboured in a galaxy-rich environment (i.e. a group or a cluster of galaxies).

\section{COMPARISON WITH RADIO-SELECTED GRG FROM LOTSS DR1}

The first selection step of our sample, i.e. the detection in hard Xray catalogues, introduces a bias towards the high accretion regime in the core of these GRGs. Indeed, the Eddington ratios of our sources fall in the range 0.01-0.37 (Ursini et al. 2018; Bassani et al. 2021). In principle, this could play a role on the evolution of their radio phase, with respect to traditional radio-selected GRG samples. While a dedicated work on the detailed comparison with a control sample of radio-selected GRG is under way, we can here compare the main properties of our HXGRGs sample with the 239 radioselected ones from LoTSS DR1 (Dabhade et al. 2020a). In Fig. 10, we compare the redshift versus radio power at $144 \mathrm{MHz}$ for the two samples (top panel), the radio power distributions (middle panel, normalized fractions), and the projected linear sizes distributions (bottom panel, normalized fractions). In addition to the LoTSS DR2 data presented in this work, we also considered the TGSS data (in the same band and at a similar resolution of $25 \mathrm{arcsec}$ ) to improve the statistics of our HXGRGs sample. In this way, we could collect the total radio powers at $\sim 150 \mathrm{GHz}$ for 18 over 23 objects of the sample, namely all HXGRGs north of $-53^{\circ}$ Dec. except the GRG candidate SWIFT J0225.8+5946, for which a redshift estimate is 

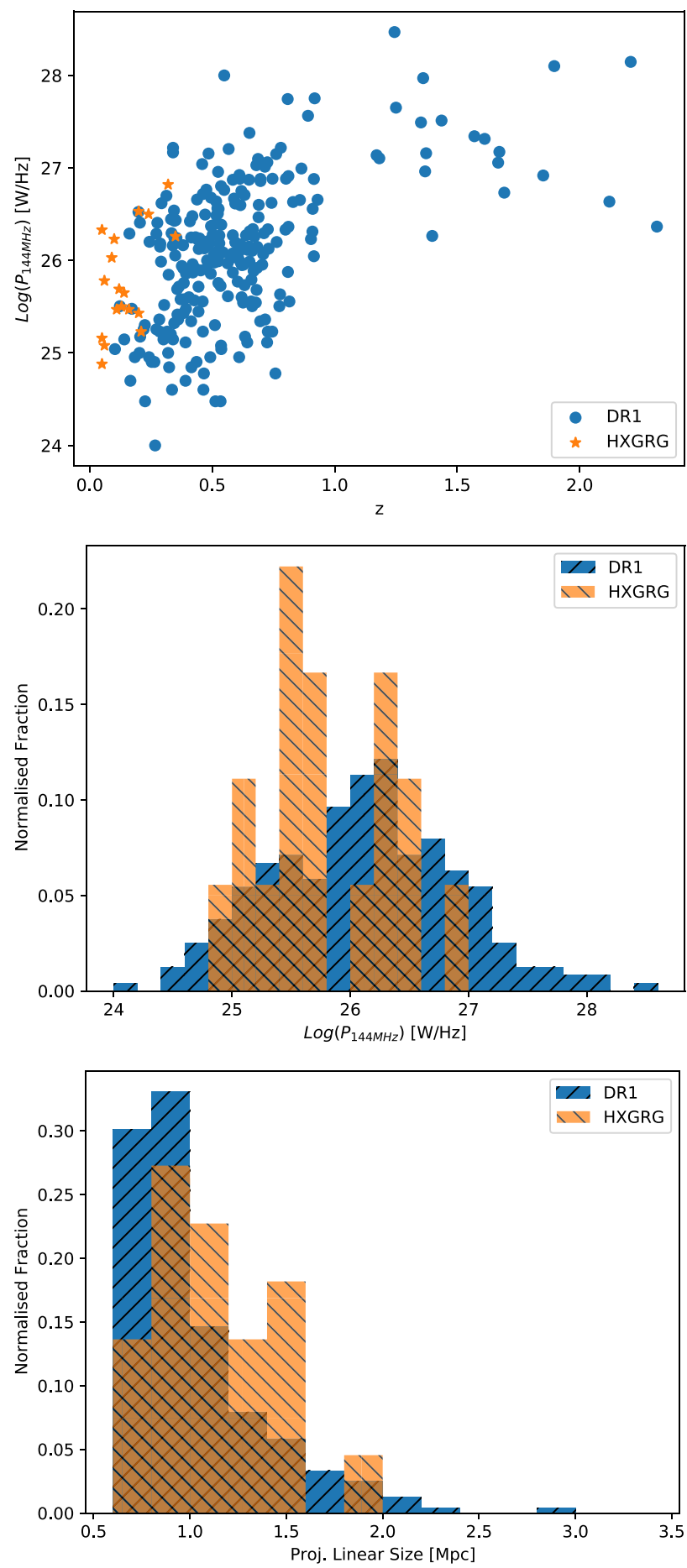

Figure 10. Comparison of the HXGRGs sample with the radio-selected GRGs from LoTSS DR1 (Dabhade et al. 2020a). Top panel: redshift versus total radio power at $144 \mathrm{MHz}$; middle panel: total radio power at $144 \mathrm{MHz}$; bottom panel: projected linear sizes.

still not available. The estimated values from TGSS, for sources not included in LoTSS DR2, are given in Table 4. For the redshift and linear size, we considered the values presented in Bruni et al. (2020) and Bassani et al. (2021), available for the full sample except SWIFT J0225.8+5946 (22 objects in total).

As visible in Fig. 10 (top panel), HXGRGs have a redshift $z$ $<0.4$, and lay in the lower envelope of the LoTSS DR1 GRGs. This is likely due to the selection criteria of the hard X-ray AGN
Table 4. Total flux density and radio power at $150 \mathrm{MHz}$ for sources from the HXGRGs sample with available TGSS data, and not included in LoTSS DR2.

\begin{tabular}{lcc}
\hline Source & $\begin{array}{c}\text { Total flux density } \\
(\mathrm{Jy})\end{array}$ & $\begin{array}{c}\text { Total radio power } \\
\log \left(\mathrm{W} \mathrm{Hz}^{-1}\right)\end{array}$ \\
\hline B3 0309+411B & $0.75 \pm 0.08$ & 25.50 \\
SWIFT J0636.5-2036 & $38.2 \pm 3.8$ & 26.33 \\
PKS 0707-35 & $8.97 \pm 0.90$ & 25.47 \\
4C 73.08 & $7.33 \pm 0.73$ & 25.78 \\
HE 1434-1600 & $0.93 \pm 0.09$ & 25.65 \\
IGR J14488-4008 & $1.41 \pm 0.14$ & 25.69 \\
IGR J17488-2338 & $2.06 \pm 0.21$ & 26.50 \\
4C + 74.26 & $6.78 \pm 0.68$ & 26.23 \\
PKS 2331-240 & $1.24 \pm 0.12$ & 24.88 \\
\hline
\end{tabular}

sample from which they are drawn. Indeed, the INTEGRAL/IBIS and Swift/BAT surveys are of relatively low sensitivity, thus preferably detecting brighter and nearby objects (typically with $z$ below $0.2-$ 0.3; see Malizia et al. 2020). The total radio powers distributions at $144 \mathrm{MHz}$ (Fig. 10, middle panel) are comparable for the two samples, with HXGRGs spanning the range $10^{25}-10^{27} \mathrm{~W} \mathrm{~Hz}^{-1}$, well within the one found for LoTSS DR1 GRGs $\left(10^{24}-10^{28.5} \mathrm{~W} \mathrm{~Hz}^{-1}\right)$. A Kolmogorov-Smirnov (KS) test on the two distributions results in a $p$-value $>10$ per cent, not allowing to discard the null hypothesis of a common parent distribution for the two. Despite the limited statistics (18 sources), this might indicate that the hard X-ray selection does not imply a difference in total radio power integrated over a Myr timescale activity, although the Eddington ratios of our GRGs fall in the high tail of the distribution for the radio-selected ones (0.01-0.37, Ursini et al. 2018; Bassani et al. 2021, to be compared with e.g. fig. 8 of Dabhade et al. 2020b). The projected linear size distributions are plotted in Fig. 10 (bottom panel) for 22 HXGRGs versus the full sample of 239 radio-selected GRGs from LoTSS DR1. The HXGRGs sample shows, on average, larger values, with a mean value of $1.15 \mathrm{Mpc}$ versus $1.03 \mathrm{Mpc}$ for the LoTSS DR2 GRGs, although the linear size range covered by the two samples is similar. A KS test confirms that the two distributions are distinct, giving a $p$-value $<$ 5 per cent for the null hypothesis. Nevertheless, an improved statistics for HXGRGs is necessary to asses this conclusion. If confirmed, this result would be consistent with the larger fraction of GRG found by Bassani et al. (2016) among hard X-ray-selected AGN. A similar result was also found by Dabhade et al. (2020b), when comparing the sizes of high-excitation giant radio galaxies (HEGRGs, i.e. objects with an efficient accretion similarly to our HXGRGs) with the ones of low-excitation giant radio galaxies (LEGRGs): on average, HEGRGs reach larger extents than LEGRGs (see fig. 7 of their work).

From a morphological point of view, Dabhade et al. (2020a) report a 5 per cent of double-double GRGs. Among the nine objects presented here, we do not have any double-double, but we can account for two clear cases among the full sample of 23 HXGRGs. This gives a fraction of $9_{-6}^{+11}$ per cent, following Poissonian statistics (Gehrels 1986), consistent within errors with the doubledouble fraction for GRGs in LoTSS DR1. Dabhade et al. (2020a) also discuss the FRI/FRII and hybrid morphology radio sources (HyMoRS) fractions (the latter being sources with a FRI morphology on one side and FRII on the other side; Saikia et al. 1996), finding 90 per cent of FRII, while $\sim 2.5$ per cent of HyMoRS. Regarding our HXGRGs sample, all sources except for one (B2 1144+35B) are FRII, resulting in a fraction of $95 \pm 5$ per cent, again compatible within errors. No cases of HyMoRS are present (as expected for small samples). Nevertheless, those authors do not provide a statistic for 
reoriented/precessing jets, X-shaped morphology, or GPS nuclei, so we postpone to a future work a full comparison of the morphological properties and restarted radio activity fraction between radio-selected and hard X-ray-selected GRGs.

Finally, we can compare the environment of the two samples, although with a limited statistics for HXGRGs. Dabhade et al. (2020a) found that at least $\sim 16$ per cent of their GRGs at $z<0.55$ are the BCG of a cluster or group of galaxies. From the analysis of our sample, 2/9 sources are found to be associated with galaxy clusters from catalogues. This gives a fraction of $22_{-14}^{+30}$ per cent, consistent within errors with what obtained for the LoTSS DR1 GRGs.

Overall, the comparison of radio power, linear size, and redshifts for the two samples suggests that the main effects of the hard Xray selection are: (1) a cut of the redshift distribution, i.e. favouring nearby objects at $z<0.4$; (2) a slightly larger growth, on average, for HXGRGs with respect to radio-selected GRG samples. No statistically significant difference in total radio power distributions of HXGRGs with respect to radio-selected GRG is found.

\section{SUMMARY AND CONCLUSIONS}

We presented LOFAR images of nine GRGs selected from hard Xray catalogues, at an angular resolution of 6 arcsec (typical rms of $150 \mu \mathrm{Jy}$ beam $^{-1}$ ) and $20 \mathrm{arcsec}$ (typical $\mathrm{rms}$ of $300 \mu \mathrm{Jy}_{\text {beam }}{ }^{-1}$ ), realized with LoTSS data. Thanks to the outstanding quality and dynamic range of LOFAR images, we could probe the complex structure of these sources, identifying for the first time diffuse, remnant plasma on the Mpc scale, off-axis emission suggesting jet reorientation/precession, and in one case an inner jet with a different axis with respect to the one connecting the lobes. In particular, for one source (B2 1144+35B), we could recover an extended, faint emission southwards of one of the lobes at a better resolution with respect to previous works (Giovannini et al. 1999), allowing us to classify this source as a fat-double. With a morphology consistent with a jet realignment, this class of sources is supposed to result from an inner accretion with a perturbed geometry, probably due to a merger. The inner jet axis detected from LOFAR images of this source, showing a different angle with respect to the most ancient and extended emission, could suggest a counter-clock jet reorientation along a Myr time scale. Similarly, another source showed a symmetrical, faint emission on a different axis with respect to the jet one, consistent with relic lobes from a previous radio phase or jet precession (J1153.9+5848). Finally, for $4 \mathrm{C}+34.47$, already showing two radio phases from our previous studies, we could confirm a discontinuous, knotty jet at a frequency an order of magnitude lower than the previous study from Hocuk \& Barthel (2010). This is suggestive of either an intermittent radio activity, or of a plasma recollimation/expansion along the jet.

An environment analysis, performed through a cross-match with four among the most recent clusters catalogue (Hao et al. 2010; Smith et al. 2012; Wen et al. 2012; Rykoff et al. 2014), allowed us to find an association with two of the presented sources: B2 1144+35B and $4 \mathrm{C}+34.47$. This was also verified through an independent environment analysis from SDSS images. In this regards, the cluster might have concurred to the fat-double morphology of source B2 $1144+35 B$, perturbing the jet axis on a Myr time-scale. On the contrary, no morphological perturbation seems to be present for $4 \mathrm{C}+34.47$. The remaining seven sources from the full HXGRGs sample falling in the footprint of the mentioned catalogues do not show a match with clusters.

Finally, we could compare the main properties of our full sample of 23 HXGRGs with the 239 radio-selected GRGs from LoTSS DR1
(Dabhade et al. 2020a). While the distributions of total radio power at $144 \mathrm{MHz}$ are consistent, a comparison of the linear sizes for the two samples suggests that HXGRGs are on average more extended than radio-selected GRGs, although with a limited statistics. This is in agreement with the findings of Bassani et al. (2016) about the parent sample of hard X-ray selected radio galaxies, from which the HXGRGs sample is drawn. Indeed, those authors found that the initial selection step would favour discovery of giant-sized radio galaxies, the latter being almost four times more abundant than in radioselected radio galaxies samples. A comparison of the environments for the HXGRGs and LoTSS DR1 GRG samples gave similar results for the two samples, with a comparable fraction of GRG matching with galaxy clusters or groups.

These results stress how the high resolution and sensitivity at the low-frequency radio regime offered by LOFAR and the LoTSS survey in particular are key to the understanding of the Myr time-scale evolution of radio galaxies, and their radio duty cycle. Ultimately, this could give significant inputs on the time-scale of the radio-mode feedback on the host galaxy (Morganti et al. 2013). In the context of the GRACE project, we are carrying out an European VLBI Network (EVN) large project from which high-resolution (pc-scale) data will shed light on the inner jet properties of these sources, particularly the ones showing a jet precession or reorientation.

\section{ACKNOWLEDGEMENTS}

The GRACE project acknowledges financial support by INAF-IAPS, in the 'Bando Nuove Idee 2019' framework. GB, FP, LB, AM, and PU acknowledge financial support under the INTEGRAL ASIINAF agreement 2019-35-HH.0. MB acknowledges support from the ERC-Stg DRANOEL, no 714245. GB and FP acknowledge support from a grant PRIN-INAF SKA-CTA 2016. AB acknowledges support from the VIDI research programme with project number 639.042.729, which is financed by the Netherlands Organisation for Scientific Research (NWO). AD acknowledges support by the BMBF Verbundforschung under the grant 05A20STA. RJvW acknowledges support from the ERC Starting Grant ClusterWeb 804208. This work is also supported by the Physics Department of Turin University (UNITO) that joined the LOFAR-IT consortium thanks to the collaboration with the Consorzio Interuniversitario per la Fisica Spaziale (CIFS) and the 'Departments of Excellence 2018-2022' Grant awarded by the Italian Ministry of Education, University and Research (MIUR) (L. 232/2016). The analysis is partially based on the OCCAM computing facility hosted by $\mathrm{C}^{3} \mathrm{~S}^{3}$ at UNITO (Aldinucci et al. 2017). This research has made use of resources provided by the Ministry of Education, Universities and Research for the grant MASF_FFABR_17_01. This investigation is supported by the National Aeronautics and Space Administration (NASA) grants GO9-20083X and GO0-21110X. LOFAR (van Haarlem et al. 2013) is the LOw Frequency ARray designed and constructed by ASTRON. It has observing, data processing, and data storage facilities in several countries, which are owned by various parties (each with their own funding sources), and are collectively operated by the ILT foundation under a joint scientific policy. The ILT resources have benefitted from the following recent major funding sources: INSU,CNRS, Observatoire de Paris and Université d'Orléans, France; BMBF, MIWF-NRW, MPG, Germany; Science Foundation Ireland (SFI), Department of Business, Enterprise and Innovation (DBEI), Ireland; NWO, The Netherlands; The Science and Technology Facilities

\footnotetext{
${ }^{3}$ http://c3s.unito.it/
} 
Council, UK; Ministry of Science and Higher Education, Poland; and Istituto Nazionale di Astrofisica (INAF), Italy. This research made use of the Dutch national e-infrastructure with support of the SURF Cooperative (e-infra 180169) and the LOFAR e-infra group. The Jülich LOFAR Long Term Archive and the German LOFAR network are both coordinated and operated by the Jülich Supercomputing Centre (JSC), and computing resources on the supercomputer JUWELS at JSC were provided 15 by the Gauss Centre for Supercomputinge e.V. (grant CHTB00) through the John von Neumann Institute for Computing (NIC). This research made use of the University of Hertfordshire high-performance computing facility and the LOFAR-UK computing facility located at the University of Hertfordshire and supported by STFC [ST/P000096/1], and of the Italian LOFAR IT computing infrastructure supported and operated by INAF, and by the Physics Department of Turin University (under an agreement with Consorzio Interuniversitario per la Fisica Spaziale) at the C3S Supercomputing Centre, Italy. The National Radio Astronomy Observatory is a facility of the National Science Foundation operated under cooperative agreement by Associated Universities, Inc. We thank the staff of the GMRT that made these observations possible. GMRT is run by the National Centre for Radio Astrophysics of the Tata Institute of Fundamental Research. This research made use of APLPY, an open-source plotting package for PYTHON (Robitaille \& Bressert 2012). This research has made use of the SIMBAD data base, operated at CDS, Strasbourg, France. The Pan-STARRS1 Surveys (PS1) and the PS1 public science archive have been made possible through contributions by the Institute for Astronomy, the University of Hawaii, the Pan-STARRS Project Office, the Max Planck Society and its participating institutes, the Max Planck Institute for Astronomy, Heidelberg and the Max Planck Institute for Extraterrestrial Physics, Garching, the Johns Hopkins University, Durham University, the University of Edinburgh, the Queen's University Belfast, the Harvard-Smithsonian Center for Astrophysics, the Las Cumbres Observatory Global Telescope Network Incorporated, the National Central University of Taiwan, the Space Telescope Science Institute, the National Aeronautics and Space Administration under Grant No. NNX08AR22G issued through the Planetary Science Division of the NASA Science Mission Directorate, the National Science Foundation Grant No. AST-1238877, the University of Maryland, Eotvos Lorand University (ELTE), the Los Alamos National Laboratory, and the Gordon and Betty Moore Foundation.

\section{DATA AVAILABILITY}

The data underlying this paper will be shared on reasonable request to the corresponding author.

\section{REFERENCES}

Ahn C. P. et al., 2012, ApJS, 203, 21

Aldinucci M., Bagnasco S., Lusso S., Pasteris P., Rabellino S., Vallero S., 2017, J. Phys.: Conf. Ser., 898, 082039

Baldi R. D., Chiaberge M., Capetti A., Rodriguez-Zaurin J., Deustua S., Sparks W. B., 2013, ApJ, 762, 30

Baldi R. D., Capetti A., Chiaberge M., Celotti A., 2014, A\&A, 567, A76

Bassani L., Venturi T., Molina M., Malizia A., Dallacasa D., Panessa F., Bazzano A., Ubertini P., 2016, MNRAS, 461, 3165

Bassani L. et al., 2021, MNRAS, 500, 3111

Begelman M. C., Blandford R. D., Rees M. J., 1980, Nature, 287, 307

Berlind A. A. et al., 2006, ApJS, 167, 1

Bock D. C. J., Large M. I., Sadler E. M., 1999, AJ, 117, 1578

Brienza M. et al., 2016, A\&A, 585, A29
Bruni G. et al., 2019, ApJ, 875, 88

Bruni G. et al., 2020, MNRAS, 494, 902

Bruzual G., Charlot S., 2011, Astrophysics Source Code Library, record ascl:1104.005

Calzetti D., Armus L., Bohlin R. C., Kinney A. L., Koornneef J., StorchiBergmann T., 2000, ApJ, 533, 682

Cantwell T. M. et al., 2020, MNRAS, 495, 143

Chambers K. C. et al., 2016, preprint (arXiv:1612.05560)

Cheung C. C., 2007, AJ, 133, 2097

Condon J. J., 1992, ARA\&A, 30, 575

Condon J. J., Cotton W. D., Greisen E. W., Yin Q. F., Perley R. A., Taylor G. B., Broderick J. J., 1998, AJ, 115, 1693

Cotton W. D. et al., 2020, MNRAS, 495, 1271

Croston J. H. et al., 2019, A\&A, 622, A10

Dabhade P. et al., 2020a, A\&A, 635, A5

Dabhade P. et al., 2020b, A\&A, 642, A153

Delhaize J. et al., 2021, MNRAS, 501, 3833

Dennett-Thorpe J., Scheuer P. A. G., Laing R. A., Bridle A. H., Pooley G. G., Reich W., 2002, MNRAS, 330, 609

Eke V. R. et al., 2004, MNRAS, 348, 866

Fruscione A. et al., 2006, in Silva D. R., Doxsey R. E., eds, Proc. SPIE Vol. 6270, Observatory Operations: Strategies, Processes, and Systems. SPIE, Bellingham, p. 62701V

Gehrels N., 1986, ApJ, 303, 336

Gillone M., Capetti A., Rossi P., 2016, A\&A, 587, A25

Giovannini G., Taylor G. B., Arbizzani E., Bondi M., Cotton W. D., Feretti L., Lara L., Venturi T., 1999, ApJ, 522, 101

Giovannini G., Giroletti M., Taylor G. B., 2007, A\&A, 474, 409

Hao J. et al., 2010, ApJS, 191, 254

Hernández-García L. et al., 2019, MNRAS, 489, 4049

Hocuk S., Barthel P. D., 2010, A\&A, 523, A9

Horton M. A., Krause M. G. H., Hardcastle M. J., 2020, MNRAS, 499, 5765

Hunik D., Jamrozy M., 2016, ApJ, 817, L1

Hurley-Walker N. et al., 2015, MNRAS, 447, 2468

Intema H. T., Jagannathan P., Mooley K. P., Frail D. A., 2017, A\&A, 598, A78

Ishwara-Chandra C. H., Saikia D. J., 1999, MNRAS, 309, 100

Jamrozy M., Konar C., Machalski J., Saikia D. J., 2008, MNRAS, 385, 1286

Jimenez-Gallardo A. et al., 2020, ApJS, 250, 7

Kharb P., Lal D. V., Merritt D., 2017, Nat. Astron., 1, 727

Konar C., Saikia D. J., Ishwara-Chandra C. H., Kulkarni V. K., 2004, MNRAS, 355,845

Krause M. G. H. et al., 2019, MNRAS, 482, 240

Kurosawa R., Proga D., 2008, ApJ, 674, 97

Kuźmicz A., Jamrozy M., 2012, MNRAS, 426, 851

Lacy M. et al., 2020, PASP, 132, 035001

Laing R. A., Riley J. M., Longair M. S., 1983, MNRAS, 204, 151

Lara L., Cotton W. D., Feretti L., Giovannini G., Marcaide J. M., Márquez I., Venturi T., 2001, A\&A, 370, 409

Liu W. et al., 2019, MNRAS, 484, 3376

Machalski J., Chyzy K. T., Jamrozy M., 2004, Acta Astron., 54, 249

Machalski J., Kozieł-Wierzbowska D., Jamrozy M., Saikia D. J., 2008, ApJ, 679,149

Mack K. H., Klein U., O’Dea C. P., Willis A. G., Saripalli L., 1998, A\&A, 329,431

McMullin J. P., Waters B., Schiebel D., Young W., Golap K., 2007, in Shaw R. A., Hill F., Bell D. J., eds, ASP Conf. Ser. Vol. 376, Astronomical Data Analysis Software and Systems XVI. Astron. Soc. Pac., San Francisco, p. 127

Malizia A., Sazonov S., Bassani L., Pian E., Beckmann V., Molina M., Mereminskiy I., Belanger G., 2020, New Astron. Rev., 90, 101545

Massaro F. et al., 2010, ApJ, 714, 589

Massaro F., Harris D. E., Cheung C. C., 2011, ApJS, 197, 24

Massaro F., D’Abrusco R., Paggi A., Masetti N., Giroletti M., Tosti G., Smith H. A., Funk S., 2013, ApJS, 209, 10

Massaro F. et al., 2015, ApJS, 220, 5

Massaro F., Álvarez-Crespo N., Capetti A., Baldi R. D., Pillitteri I., Campana R., Paggi A., 2019, ApJS, 240, 20 
Massaro F. et al., 2020a, ApJS, 247, 71

Massaro F., Capetti A., Paggi A., Baldi R. D., Tramacere A., Pillitteri I., Campana R., 2020b, ApJ, 900, L34

Moore B., Frenk C. S., White S. D. M., 1993, MNRAS, 261, 827

Morganti R., Fogasy J., Paragi Z., Oosterloo T., Orienti M., 2013, Science, 341, 1082

Natarajan P., Pringle J. E., 1998, ApJ, 506, L97

O’Dea C. P., 1998, PASP, 110, 493

Offringa A. R., Smirnov O., 2017, MNRAS, 471, 301

Offringa A. R. et al., 2014, MNRAS, 444, 606

Orrù E. et al., 2015, A\&A, 584, A112

Robitaille T., Bressert E., 2012, Astrophysics Source Code Library, record ascl: 1208.017

Rykoff E. S. et al., 2014, ApJ, 785, 104

Saikia D. J., Thomasson P., Jackson N., Salter C. J., Junor W., 1996, MNRAS, 282, 837

Saripalli L., Roberts D. H., 2018, ApJ, 852, 48

Saripalli L., Malarecki J. M., Subrahmanyan R., Jones D. H., Staveley-Smith L., 2013, MNRAS, 436, 690

Schlafly E. F., Meisner A. M., Green G. M., 2019, ApJS, 240, 30

Schoenmakers A. P., Mack K. H., de Bruyn A. G., Röttgering H. J. A., Klein U., van der Laan H., 2000, A\&AS, 146, 293

Shimwell T. W. et al., 2017, A\&A, 598, A104

Shimwell T. W. et al., 2019, A\&A, 622, A1

Shulevski A. et al., 2019, A\&A, 628, A69

Smith A. G., Hopkins A. M., Hunstead R. W., Pimbblet K. A., 2012, MNRAS, 422,25

Stassun K. G. et al., 2019, AJ, 158, 138

Stern D. et al., 2012, ApJ, 753, 30

Stuardi C. et al., 2018, ApJS, 235, 32

Stuardi C. et al., 2020, A\&A, 638, A48
Subrahmanyan R., Hunstead R. W., Cox N. L. J., McIntyre V., 2006, ApJ, 636,172

Tasse C. et al., 2018, A\&A, 611, A87

Tasse C. et al., 2020, preprint (arXiv:2011.08328)

Ursini F. et al., 2018, MNRAS, 481, 4250

van Haarlem M. P. et al., 2013, A\&A, 556, A2

van Weeren R. J. et al., 2020, preprint (arXiv:2011.02387)

Vasconcellos E. C., de Carvalho R. R., Gal R. R., LaBarbera F. L., Capelato H. V., Frago Campos Velho H., Trevisan M., Ruiz R. S. R., 2011, AJ, 141, 189

Wen Z. L., Han J. L., Liu F. S., 2012, ApJS, 199, 34

Wenger M. et al., 2000, A\&AS, 143, 9

White R. L., Becker R. H., Helfand D. J., Gregg M. D., 1997, ApJ, 475, 479

Willis A. G., Strom R. G., Wilson A. S., 1974, Nature, 250, 625

\section{APPENDIX A: THE X-RAY DETECTION OF THE RADIO JET IN $4 \mathrm{C}+\mathbf{3 4 . 4 7}$}

We searched in the Chandra archive and discovered that $4 \mathrm{C}+34.47$, lying in the field of CSWA 14 (obs. ID 18224), was serendipitously observed in the X-rays in 2016 September. We retrieved the data set from the Chandra archive, and performed the data reduction and analysis following the same procedures described in the Chandra Interactive Analysis of Observations (CIAO; Fruscione et al. 2006) threads using CIAO v4.11 and Chandra Calibration Database v4.8.4. We found that the X-ray counterpart of $4 \mathrm{C}+34.47$ lies on the edge of the Advanced CCD Imaging Spectrometer-S (ACIS-S) chip 3, a few arcminutes angular separation from the aim point, while the northern lobe is in the gap between different chips. However, overlaying radio

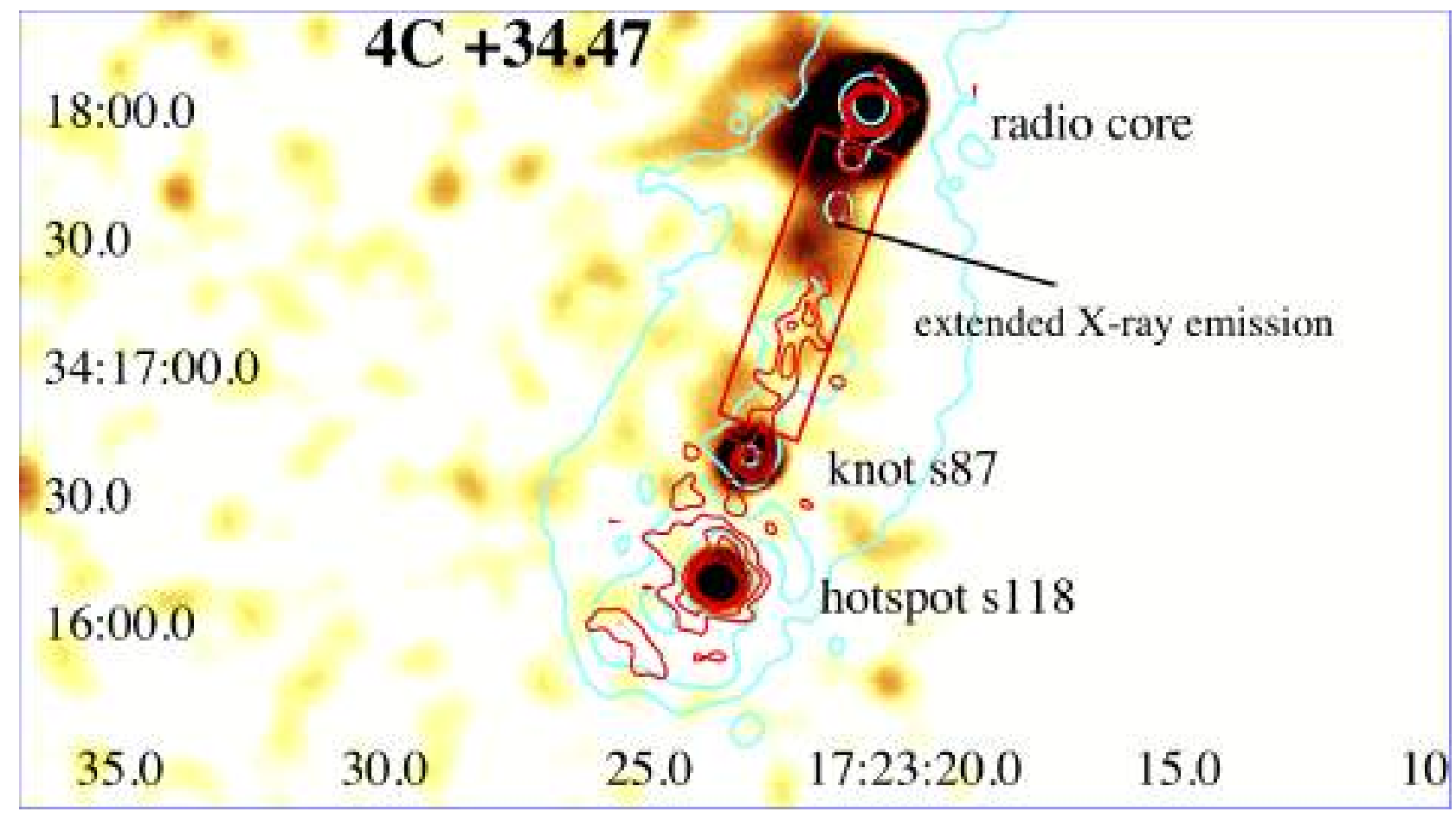

Figure A1. Chandra image in the $0.5-7 \mathrm{keV}$ energy range (exposure corrected and with the readout streak removed) of $4 \mathrm{C}+34.47$ with $1.4 \mathrm{GHz}$ radio contours overlaid in red and those at $150 \mathrm{MHZ}$ obtained from the LOFAR observation shown in cyan. Low-frequency radio contours are drawn starting from the level of $0.002 \mathrm{mJy}_{\text {beam }}^{-1}$ increasing by a factor of 4 , while those at $1.4 \mathrm{GHz}$ start at $0.0001 \mathrm{mJy} \mathrm{beam}^{-1}$ increasing by a factor of 2 and reach $0.032 \mathrm{mJy}$ beam ${ }^{-1}$. The $\mathrm{X}$-ray image is smoothed with a Gaussian kernel of size equal to $7.872 \mathrm{arcsec}$ and pixels have size large as twice native pixel size of Chandra (i.e. 0.984 arcsec). It is quite evident the X-ray detection of the counterpart of both the knot s87 and the hotspot s118 and the presence of extended X-ray emission between the position of the radio core of $4 \mathrm{C}+34.47$, lying at the edge of the chip, and the $\mathrm{s} 87$ jet knot. All high-energy counterparts of these radio structures are detected at level of significance higher than $5 \sigma$ Gaussian equivalent. 
contours to the X-ray image, we discovered the X-ray counterpart of the brightest knot and the hotspot in the southern lobe, at 87 and 118 arcsec angular separation from the radio core, respectively. These radio components are detected in $0.5-7 \mathrm{keV}$ energy range at level of significance greater than $5 \sigma$ Gaussian equivalent.

Adopting the nomenclature proposed in Massaro, Harris \& Cheung (2011), we labelled the first radio feature as s87 while the hotspot as s118. We measured 70 and $48 \mathrm{X}$-ray photons in the $0.5-7 \mathrm{keV}$ energy range for $\mathrm{s} 87$ and $\mathrm{s} 118$, respectively. We also claim the detection of extended X-ray emission along the radio axis, in the southern direction, located between the radio core and the knot s87 with $153 \mathrm{X}$-ray photons located within the red rectangular region shown in Fig. A1, and correspondent to a detection of $>5 \sigma$ level of confidence.

In Fig. A1, we show the Chandra $0.5-7 \mathrm{keV}$ flux map, exposure corrected and with radio contours overlaid, drawn from the $1.4 \mathrm{GHz}$ radio map retrieved from the historical archive of the $\mathrm{VLA}^{4}$ and shown together with those at $144 \mathrm{MHz}$ one obtained with LOFAR. The Chandra flux map has been built adopting the same procedure adopted for the 3CR Chandra Snapshot Survey (see e.g. Massaro et al. 2010, 2013, 2015; Stuardi et al. 2018; Jimenez-Gallardo et al. 2020 for additional details), as well as adopting the FLUXIMAGE task of CIAO. Finally, we highlight that the readout streak, present in the Chandra observation given the relatively high flux of the X-ray nucleus, was also removed in the image shown in Fig. A1 according to the standard procedure..$^{5}$

\section{APPENDIX B: SERENDIPITOUS DISCOVERY OF A RADIO GALAXY}

During inspection of the sky region in the LoTSS image of GRG J0801.7+4764, we found a new radio source that we name J0802+4711. As evident in the LOFAR image (see Fig. B1), the source displays two sets of lobes: an internal dimmer one, and an external brighter one (thus resembling the morphology of a doubledouble radio galaxy). No core is visible at this frequency in between the two set of lobes. The $144 \mathrm{MHz}$ flux density of each individual lobe is reported in Table B1. The source extent is roughly 2 arcmin, implying a projected linear size smaller than the GRG threshold of $0.7 \mathrm{Mpc}$ for a redshift up to $z=0.45$. The source is detected in the Faint Images of the Radio Sky at Twenty-cm (FIRST) and VLASS surveys, where however only the core and external couple of lobes are visible (i.e. the brightest and compact emission in the $\mathrm{GHz}$ domain, see Fig. B1). Finally, the NVSS survey resolves the source into only two components, due to the lower angular resolution (45 arcsec), corresponding to the external+internal lobes regions. Nevertheless, it allows us to estimate a total flux density at $1.4 \mathrm{GHz}$ of $62 \mathrm{mJy}$. The flux density values extracted for the single components in the LOFAR, FIRST, and VLASS surveys are reported in Table B1. In the FIRST image, the core is detected at only $\sim 5 \sigma$ significance $\left(0.76 \pm 0.08 \mathrm{mJy}\right.$ beam $^{-1}$, with an $\mathrm{rms}$ of $\left.0.15 \mathrm{mJy} \mathrm{beam}^{-1}\right)$, and thus it is not reported in the FIRST sources catalogue. Nevertheless, its position at the centre of a large and complex radio source makes the detection more reliable. The location of this central component is RA $(\mathrm{J} 2000)=08: 02: 43.994$ and Dec. $(\mathrm{J} 2000)=+47: 11: 37.325$

\footnotetext{
${ }^{4}$ http://archive.nrao.edu/nvas/

${ }^{5}$ https://cxc.cfa.harvard.edu/ciao/threads/acisreadcorr/
}

with a positional uncertainty of around $1.0 \operatorname{arcsec}^{6}$ In the LOFAR image at 6 arcsec the core is not distinguishable, its position from FIRST corresponding to a diffuse, extended emission region between the lobes. This results in a plateau of flux density that makes an upper limit estimate not feasible. At higher frequencies ( $3 \mathrm{GHz}$ ), the core is visible in the VLASS survey, with a $10 \sigma$ detection in a position correspondent to the one from FIRST. Its flux density, extracted via Gaussian fitting, is of $2.88 \pm 0.43 \mathrm{mJy}$. This allows us to obtain a first indication of the spectral index between $1.4 \mathrm{GHz}$ and $3 \mathrm{GHz}$ of $\alpha=1.69$, indicating an inverted spectrum typical of AGN core components. Nevertheless, the different resolution between the two surveys, and the possibility of a variable flux density for the core, makes this an approximate estimate only, to be considered with caution. The external couple of lobes is visible as well, although only the most compact regions (hotspots), while the internal lobes are not detected. The fact that the external lobes hotspots are visible at $3 \mathrm{GHz}$ suggests that they have been recently replenished with plasma from the jets, implying that these lobes are the ones linked to the ongoing radio phase. In this case, the internal couple of lobes should be older, or resulting from a lateral expansion of the jet due to a lower density of the medium. Further data are anyway needed to test this hypothesis.

Within the FIRST positional error circle, we found only one optical source clearly detected in the SDSS Sky image. The source is stellar like in the image, and quite dim, with magnitude ranging from 19.4 in $z$ to 21.5 in $u$, and is classified as a star in the SDSS catalogue (Vasconcellos et al. 2011), as well as in the recently release of the Transiting Exoplanet Survey Satellite (TESS) catalogue, incorporating also Gaia Data Release 2 (Stassun et al. 2019). The source is also reported in the unWISE catalogue, containing the positions and fluxes of roughly 2 billion objects observed by the Wide-field Infrared Survey Explorer (WISE) over the full sky (Schlafly, Meisner \& Green 2019). Fluxes in W1 and W2 bands have been converted into Vega magnitudes following the prescription given in the catalogue: we obtained a W1 and W2 magnitudes of 15.58 and 14.41, respectively, which provide a W1-W2 colour of 1.17 , above the line to classify the source as a possible AGN (see e.g. Stern et al. 2012). Finally, we note that an infrared source is also reported in the AllWISE catalogue but with a position offset by 3.2 arcsec, and with brighter W1 and W2 magnitudes (difference of 1.2 and $0.5 \mathrm{mag}$ in $\mathrm{W} 1$ and $\mathrm{W} 2$, respectively); it is possible that this is the same unWISE source discussed above, but better located, and with slightly different fluxes after co-addition of all publicly available 3-5 $\mu \mathrm{m}$ WISE images (and a more sophisticated data analysis).

In order to estimate the photometric redshift of the source, we built the optical-infrared spectral energy distribution (SED) by using its optical SDSS magnitudes $(u, g, r, i, z)$ and infrared WISE ones (W1 and W2, see Fig. B2). We used the 2SPD code, developed by Baldi et al. (2013) and updated by Baldi et al. (2014), to model the optical-infrared SED. This code can include in the SED fit: two stellar populations of different ages, a dust component with a single (or two) temperature blackbody emission(s), and an AGN spectrum with emission lines. The code searches for the best match between the total (stellar+dust+AGN) models and the photometric points by minimizing the appropriate $\chi^{2}$ function (see Baldi et al. 2013 for details). The main parameters that 2 SPD returns are the photometric redshift and the stellar age of the host. To estimate the uncertainties

${ }^{6}$ To estimate the position error we have used the empirical expression given in White et al. (1997); the value we obtain is in agreement with the values quoted for point sources at the detection limit of the FIRST catalogue. 


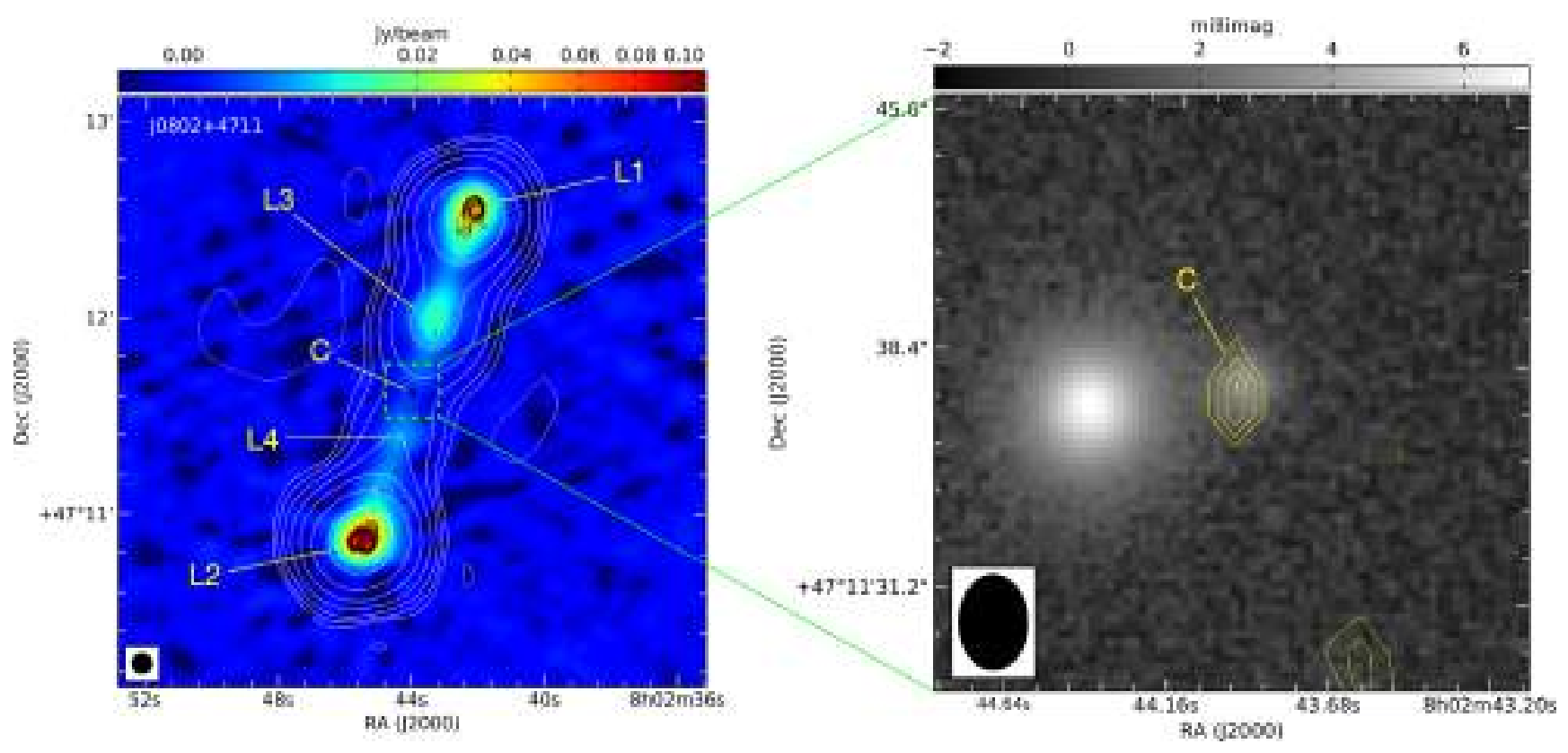

Figure B1. Left-hand panel: LOFAR image at 6 arcsec resolution of the newly discovered RG J0802+4711. Black contours are from VLASS, and correspond to rms multiples, namely $0.16 \times(-3,3,4,5,6,7,8,9,10,15,20,30,40,50,100)$. White contours are from the LOFAR image at 20 arcsec resolution, and corresponds to $3 \sigma$ multiples, namely $3 \sigma \times(-1,1,2,4,8,16,32,64,128,256)$. The different components are indicated as follows: northern external lobe (L1); southern external lobe (L2); northern internal lobe (L3); southern internal lobe (L4); and the core (C). The LOFAR 6 arcsec beam is shown in the lower left-hand corner. Right-hand panel: zoom on the core region, showing an overlay of the Pan-STARRS $i$-band image (in grey scale) and VLASS (in contours). Contours are multiples of the VLASS image rms, namely $\mathrm{rms} \times(-3,3,4,5,6,7,8,9,10)$. Negative contours are dashed. The VLASS beam is shown in the lower left-hand corner.

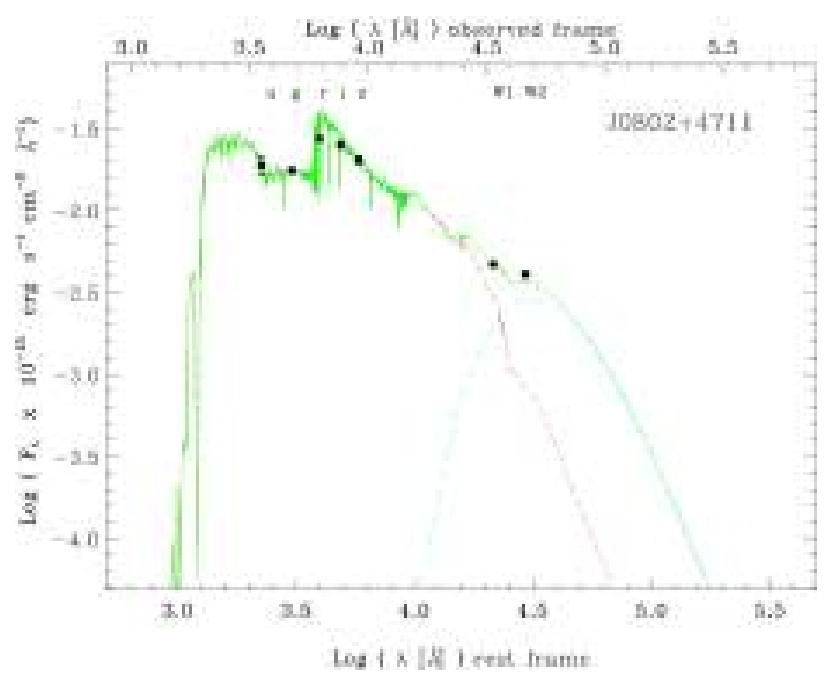

Figure B2. Optical-infrared (SDSS-WISE) SED of the galaxy J0802+471. The photometric points are fit with the 2SPD fitting code (Baldi et al. 2013, 2014): a 0.3 Gyr old stellar population is the red (dot-dashed) line, the dust blackbody component is the green (dashed) lines, and the total model is the green solid line. The resulting photometric redshift of the source is $0.57_{-0.29}^{+0.10}$ The wavelengths on the top of the plot correspond to observed wavelengths, while those on the bottom are in the rest frame.

on the photo- $z$ derivation, we measure the 99 per cent confidence solutions. We adopt the synthetic stellar templates from Bruzual \& Charlot (2011), with single stellar populations with ages ranging from $1 \mathrm{Myr}$ to $12.5 \mathrm{Gyr}$. We also use a dust-screen model for the extinction normalized with the free parameter $A_{V}$, and the Calzetti et al. (2000) extinction law. We run the 2 SPD code for the SED of
Table B1. Radio flux densities at $144 \mathrm{MHz}$ for the different source components of the newly discovered radio galaxy. The last column report the spectral index for the available VLA surveys in the same configuration (FIRST and VLASS), and at similar resolution.

\begin{tabular}{lcccc}
\hline Component & $\begin{array}{c}\text { LOFAR } \\
(\mathrm{mJy})\end{array}$ & $\begin{array}{c}\text { FIRST } \\
(\mathrm{mJy})\end{array}$ & $\begin{array}{c}\text { VLASS } \\
(\mathrm{mJy})\end{array}$ & $\alpha_{1.4}^{3}$ \\
\hline Core & - & $0.79 \pm 0.08$ & $2.88 \pm 0.43$ & 1.69 \\
North lobe (int) & $54 \pm 11$ & $<0.45$ & $<0.48$ & - \\
South lobe (int) & $15 \pm 3$ & $<0.45$ & $<0.48$ & - \\
North lobe (ext) & $170 \pm 30$ & $14.2 \pm 1.4$ & $8.20 \pm 1.23$ & -0.72 \\
South lobe (ext) & $270 \pm 50$ & $32.5 \pm 3.2$ & $16.2 \pm 2.4$ & -0.91 \\
\hline
\end{tabular}

J0802+4711 by including a single stellar population and a single dust component, which are necessary to reproduce, respectively, the optical and infrared part of the SED. The best fit is consistent with a $0.3 \mathrm{Gyr}$ old stellar population (extincted $A_{V}=0.9$ ). The derived photometric redshift is $0.57_{-0.29}^{+0.10}$. The total radio power at $1.4 \mathrm{GHz}$, calculated using the nominal value of the estimated redshift, is $P_{1.4 \mathrm{GHz}}=5.0 \times 10^{25} \mathrm{~W} \mathrm{~Hz}^{-1}$. Even considering the redshift uncertainty, this would place the source in the radio-loud regime according to Condon (1992), who defines a threshold of $10^{23} \mathrm{~W} \mathrm{~Hz}^{-1}$ to distinguish the radio-quiet and radio-loud populations. The angular size as calculated from the LOFAR 20 arcsec image is 160 arcsec, which would translate into a projected linear size of $1040 \mathrm{kpc}$ considering the nominal value of the photometric redshift. This would be above the conventional threshold for GRG (700 kpc). However, considering the large redshift uncertainty, the projected linear size could still be as low as $670 \mathrm{kpc}$. Thus, we cannot consider this object a bona fide GRG until a more precise redshift estimate will be available. 
Concluding, we found a possible AGN detected at radio, optical, and infrared wavebands, whose nature is still uncertain, and cannot be clarified until the source is either detected in X-rays, or spectroscopically classified. Unfortunately, the source has not been observed at X-ray wavelengths yet, nor it is bright enough for an optical spectroscopic follow-up with small telescopes (more easily available). For this reason, we plan to perform a Swift/XRay Telescope (XRT) observation in the near future. Further radio observations will shed light on this intriguing source.

This paper has been typeset from a $\mathrm{T}_{\mathrm{E}} \mathrm{X} / \mathrm{L} \mathrm{T} \mathrm{T}_{\mathrm{E}} \mathrm{X}$ file prepared by the author. 